\title{
An adaptive discontinuous Galerkin method for the simulation of hurricane storm surge
}

\author{
Nicole Beisiegel ${ }^{1,2}$ (D) $\cdot$ Stefan Vater ${ }^{3} \cdot$ Jörn Behrens ${ }^{4,5} \cdot$ Frédéric Dias $^{1,2,6}$ \\ Received: 3 September 2019 / Accepted: 29 January 2020 / Published online: 21 March 2020 \\ (C) The Author(s) 2020
}

\begin{abstract}
Numerical simulations based on solving the 2D shallow water equations using a discontinuous Galerkin (DG) discretisation have evolved to be a viable tool for many geophysical applications. In the context of flood modelling, however, they have not yet been methodologically studied to a large extent. Systematic model testing is non-trivial as no comprehensive collection of numerical test cases exists to ensure the correctness of the implementation. Hence, the first part of this manuscript aims at collecting test cases from the literature that are generally useful for storm surge modellers and can be used to benchmark codes. On geographic scale, hurricane storm surge can be interpreted as a localised phenomenon making it ideally suited for adaptive mesh refinement (AMR). Past studies employing dynamic AMR have exclusively focused on nested meshes. For that reason, we have developed a DG storm surge model on a triangular and dynamically adaptive mesh. In order to increase computational efficiency, the refinement is driven by physics-based refinement indicators capturing major model sensitivities. Using idealised numerical test cases, we demonstrate the model's ability to correctly represent all source terms and reproduce known variability of coastal flooding with respect to hurricane characteristics such as size and approach speed. Finally, the adaptive mesh significantly reduces computing time with no effect on storm waves measured at discrete wave gauges just off the coast which shows the model's potential for use as a robust simulation tool for real-time predictions.
\end{abstract}

Keywords Storm surges · Discontinuous Galerkin · Wind stress · Wind drag · Adaptive mesh refinement · Shallow water equations

\section{Introduction}

Extreme storms and floods are among the most devastating natural disasters worldwide faced by inhabitants of coastal areas and those whose livelihood depends on marine resources alike (see, e.g. (Lin et al. 2010)). Their accurate simulation and

Responsible Editor: Eric Deleersnijder

Nicole Beisiegel

nicole.beisiegel@ucd.ie

1 Earth Institute, University College Dublin, Dublin, Ireland

2 School of Mathematics \& Statistics, University College Dublin, Dublin, Ireland

3 Institute of Mathematics, Freie Universität Berlin, Berlin, Germany

4 Department of Mathematics, Universität Hamburg, Hamburg, Germany

5 Center for Earth System Research and Sustainability, Universität Hamburg, Hamburg, Germany

6 ENS Paris-Saclay, CNRS, Université Paris-Saclay, Centre Borelli, 94235 Cachan, France prediction are vital for hazard assessment and the implementation of mitigation strategies such as the opening and closing of flood barriers in order to protect local populations and property. Due to the time critical nature of forecasts, current operational simulation environments that form the basis of flood forecasts are chosen for their robustness and optimised run time. However, this efficiency comes at the cost of having to use reduced hydrodynamical model approximations for the underlying processes and simplified parameterisations for physical forcing terms. This means that operational models often do not model the full physics (Tull 2018). Instead, such simple inundation models simply raise water levels to a given constant elevation. Linear approaches like this are computationally inexpensive and work well for generalised scenarios and at broad geographic scale, but as we become more specific and local in the attempted projections, the complexities increase, and non-linearities become more significant in the behaviour of the modelled physical processes. Therefore, we cannot use linear mathematical approaches to precisely model these situations. Non-linear models, on the other hand, can be costly, and the high-order information they contain might require advanced filtering to guarantee numerical robustness and efficiency. These more accurate models, however, are of 
critical importance. As was shown in (Barnard et al. 2019), the accuracy of projections for future events, in particular with respect to our changing climate, depends on the accurate modelling of small scale physical dynamics at large scale.

In this study, we focus on discontinuous Galerkin (DG) methods to solve the 2D non-linear shallow water equations. These depth integrated equations are computationally less demanding than more accurate 3D Navier-Stokes equations and have been shown to yield good results for coastal applications. Since realistic measurement data often lacks high-order smoothness, and we aim to build a model that will eventually be useful for practical applications, we furthermore concentrate on piecewise linear polynomials to represent our quantities of interest. This leads to a formally second order in space accurate numerical scheme which we believe to be sufficient to be useful. DG methods have recently gained a lot of attention for geophysical applications because of their local mass conservation and geometric flexibility. Moreover, numerical computations are performed locally since elements only communicate over element interfaces (edges) through the computation of numerical fluxes. This is a particular advantage for current state-of-the-art computational architectures and although beyond the scope of this study, we remark that the local nature of this discretisation makes it ideal for parallelisation as shown for example in (Abdi et al. 2019).

Due to their relative computational expense, current operational flood models do not employ DG discretisations; they are based on other numerical techniques (see also Danilov 2013). A common choice is continuous finite elements (Piggott et al. 2008a), because they work well with nonuniform meshes. However, they only yield global mass conservation and parallelisation is more difficult because large linear systems often need to be inverted. The majority of computer models used in practice rely on finite difference discretisations. Those have the advantage of discretisations for parameterisations of source terms such as bottom friction being existent and well established. We remark, though, that in theory, the implementation of parameterisations in a DG framework would be easy as it offers the opportunity to control in cell values as opposed to point values only and it does not formally require differentiability of the source term. In addition to representing complex coastlines by the use of curvilinear meshes - an approach that has been successfully used in finite difference frameworks-DG methods offer the possibility to represent varying geometries directly through highorder approximations within elements. Another common choice for flood modelling are finite volume (FV) methods which are conservative and work well even on unstructured grids as shown in (Danilov 2013). First-order DG methods are equivalent to FV methods, so that DG methods can be seen as one possibility to extend FV methods to higher-order accuracy without requiring high-order reconstructions that might be computationally expensive.
A higher computational efficiency of mesh-based numerical methods such as DG methods can be achieved by using nonuniform meshes (Piggott et al. 2008b) or dynamical adaptive mesh refinement as in (Behrens et al. 2005), leading to an improved performance on current computing architectures. DG methods have been successfully employed in combination with a non-uniform but static mesh to hindcast the coastal flooding caused by hurricane Ike (Dawson et al. 2011). Ideally, and to save computational time, the use of dynamically adaptive nonuniform meshes would be preferable. These meshes are usually driven by either heuristic (or physics-based) refinement indicators or error estimators that are based on model sensitivities which in turn can be derived from solving adjoint equations, as in (Farrell et al. 2013) for a finite element framework. Solving adjoint equations, however, can be computationally expensive as it requires the solution of a different set of equations backwards in time with coefficients that result from the forward solution of the system. This increases memory requirements significantly. Additionally, Beckers et al. (2019) show that the discontinuous nature of DG methods poses systematic difficulties on deriving appropriate adjoint equations. The additional flexibility of a dynamically adaptive mesh would decrease the dependence of a high level of mesh optimisation that is particular for the geographic region of interest. An approach using finite volumes on a quadrilateral nested mesh has been used in one previous study (Mandli and Dawson 2014), but the full potential of dynamically adaptive mesh refinement using physics-based refinement indicators for fully adaptive meshes is yet to be explored. Hence, this study aims at investigating DG methods for flood applications on a dynamically adaptive triangular mesh.

The accurate modelling of inundation using the shallow water equations is mathematically challenging as the interface between water and land becomes a moving boundary, and the theoretical validity of the underlying equations breaks down in near-dry regions. Recently developed numerical methods show improved robustness due to improved limiting and filtering techniques (Vater et al. 2015; Vater et al. 2019). We will adopt the novel limiting strategy presented in (Vater et al. 2019) which features a velocity-based reconstruction of the momentum and allows us to compute meaningful velocities even close to the wet/dry interface.

The major driving forces of storm surges are extreme winds and pressure gradients. This study considers hurricane storm surges in Sect. 4.1 and employs the cyclonic wind model by (Holland 1980) to compute continuous wind fields as well as corresponding atmospheric pressures. Finally, we implement all source terms that are relevant for coastal flooding and storm waves and show that in combination with the dynamically adaptive mesh refinement as proposed in (Behrens et al. 2005), we built a new model that is suitable for coastal storm surge modelling.

In summary, in this study, we combine a DG model with a dynamically adaptive mesh that is driven by physics-based 
refinement indicators. We show that a recently developed advanced limiting strategy to treat wetting and drying as well as the discrete implementation of all relevant source terms lead to robust and accurate results on this mesh.

This paper is organised as follows. Section 2 outlines the numerical model and summarises the implementation of all relevant source terms, and Sect. 2.2 gives a brief introduction to the adaptive mesh refinement used later on. The results section is separated into two sections: Section 3 presents a number of numerical test cases ranging from analytical to idealised yet realistic scenarios that showcase the model's inundation stability, conservation properties, as well as the robustness of the wind forcing. In Sect. 4, we then investigate the described model using the adaptive mesh refinement from Sect. 2.2 to demonstrate its suitability for idealised storm surge modelling on a dynamically adaptive mesh. The Sect. 5 gives an outlook for future work and discusses implications, shortfalls and limitations of the study.

\section{A discontinuous Galerkin discretisation of the full 2D non-linear shallow water equations}

The system of equations of interest for this study are depthintegrated shallow water equations in two dimensions which can be written in flux form

$\frac{\partial \mathbf{U}}{\partial t}+\nabla \cdot \mathbf{F}(\mathbf{U})=\mathbf{S}(\mathbf{U}) \quad$ in $\quad \Omega \times T$,

where the prognostic variables are $\mathbf{U}=(h, h \mathbf{u})^{\top}$ : the water depth $h$ and the 2D momentum $h \mathbf{u}$ with a $2 \mathrm{D}$ velocity $\mathbf{u}=(u, v)^{\top}$ defined on $\Omega \times T$, with $\Omega \subset \mathbb{R}^{2}$ and $T$ a finite time interval. Spatial coordinates are denoted as $\boldsymbol{x}=(x, y)^{\top} \in \Omega$. The partial temporal derivative is denoted by $\frac{\partial \mathbf{U}}{\partial t}$ and $\nabla \cdot:=\left(\frac{\partial}{\partial x}, \frac{\partial}{\partial y}\right)$. is the 2D divergence operator. The flux $\mathbf{F}$ and source term $\mathbf{S}$ are defined as

$\mathbf{F}(\mathbf{U})=\left[\begin{array}{c}h \mathbf{u}_{g} \\ h \mathbf{u} \otimes \mathbf{u}+\frac{g}{2} h^{2} \mathbf{I}_{2}\end{array}\right]$,

$\mathbf{S}(\mathbf{U})=-\left[\begin{array}{c}0 \\ g h \nabla b+\boldsymbol{\tau}_{C}-\rho^{-1}\left(\boldsymbol{\tau}_{W}+h \nabla p_{A}+\boldsymbol{\tau}_{B}\right)\end{array}\right]$

where $g=9.81 \mathrm{~m} \mathrm{~s}^{-2}$ is the acceleration due to gravity and $\mathbf{I}_{2}$ is the $2 \times 2$ identity matrix. We denote the temporally constant bathymetry by $b=b(\mathbf{x})$ and introduce the notation $H(\mathbf{x}, t)=$ $h(\mathbf{x}, t)+b(\mathbf{x})$ for the total height (see also Fig. 1). In addition to the influence of bathymetry, the source term $\mathbf{S}$ comprises a Coriolis forcing $\tau_{C}$, a vector-valued wind stress $\tau_{W}$ and the water density $\rho$, which we will assume to be constant throughout this study, as well as the atmospheric pressure $p_{A}$ and a bottom

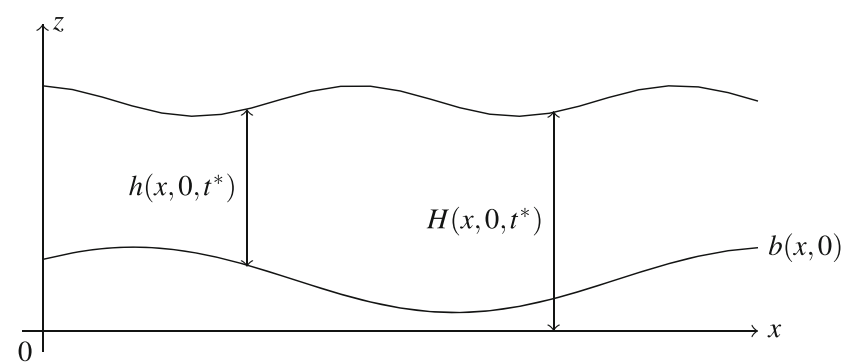

Fig. 1 2D shallow water equations: sketch of variables $h, H$ and $b$, projection onto $y=0$ at time $t=t^{*}$. Here, $z$ is an upward pointing vertical coordinate

friction $\tau_{B}$ which are described in more detail in Sect. 2.1. Throughout this paper, vector-valued quantities are indicated by a bold print while all other quantities are assumed to be scalar.

It is well understood that the shallow water approximation is somewhat over simplified for applications of coastal inundation, where non-hydrostatic effects may very well play a significant role. On the other hand, many practical applications involve large uncertainties in data and parameterisations of small-scale effects can be calibrated to yield practically useful results. Furthermore, a very effective extension of the shallow water solver by nonhydrostatic correction following Casulli and Stelling (1998) can be applied to our discretisation (see Jeschke et al. 2017).

We discretise Eq. (1) using a discontinuous Galerkin approach comprised of (a) decomposing the domain $\Omega=\sum_{i} \Omega_{i}$ into triangles, (b) approximating $\mathbf{U}=\sum_{k} \mathbf{U}_{k}(t) \phi_{k}(x)$ by linear Lagrange polynomials locally in each triangle and (c) integrating locally in space against test functions. Our test functions are linear Lagrange polynomials, so that the resulting semidiscrete system reads

$\int_{\Omega_{i}} \mathbf{U}_{t} \phi_{j} \mathrm{~d} \mathbf{x}+\int_{\Omega_{i}} \nabla \cdot \mathbf{F}(\mathbf{U}) \phi_{j} \mathrm{~d} \boldsymbol{x}+\int_{\partial \Omega_{i}}\left(\mathbf{F}^{*}(\mathbf{U})-\mathbf{F}(\mathbf{U})\right) \cdot \mathbf{n} \phi_{j} \mathrm{~d} \mathbf{S}=\int_{\Omega_{i}} \mathbf{S}(\mathbf{U}) \phi_{j} \mathrm{~d} \mathbf{x}$.

In Eq. (3), $\mathbf{F}^{*}$ is a numerical Rusanov flux at the cell interfaces defined as

$\mathbf{F}^{*}=\frac{\mathbf{F}\left(\mathbf{U}_{\mathrm{L}}\right)+\mathbf{F}\left(\mathbf{U}_{\mathrm{R}}\right)}{2}-\lambda_{\max }\left(\mathbf{U}_{\mathrm{R}}-\mathbf{U}_{\mathrm{L}}\right)$,

where $\mathbf{U}_{\mathbf{L}}$ and $\mathbf{U}_{\mathbf{R}}$ are the prognostic variables on the left and right of the respective edge and $\lambda_{\max }=\max (|u|+\sqrt{g h},|v|$ $+\sqrt{g h})$ is the maximum local directional shallow water wave speed, see also (Toro 2009). As demonstrated in (Toro 2009), other choices to compute $\mathbf{F}^{*}$ are available. In this study, we chose the Rusanov flux for its computational simplicity and its slightly higher dissipation compared to the computation of the exact solution of the Riemann problem (see (LeVeque 2002)) which we believe adds to the robustness of the presented DG method. Note that we integrated the flux integral by parts twice to obtain the often called strong form (Hesthaven and Warburton 2008). This form has desirable properties with respect to well-balancing 
as shown in (Beisiegel 2014) and elaborated in more detail in Sect. 2.1.3. We solve the integrals in Eq. (3) with an exact, i.e. third-order, interpolatory Gauss quadrature with corresponding Gauss-Legendre points in order to not introduce lower order numerical errors into the scheme.

This allows to extract the time derivative and, after re-organisation, write the system (Eq. (3)) as a system of equations in $t$ of the form

$$
\frac{d \mathbf{U}_{k}}{d t}=\mathbf{H}\left(\mathbf{U}_{k}\right)
$$

where $\mathbf{H}$ denotes the discretised flux and source terms. This system (Eq. (4)) can be solved using a strong-stability preserving (SSP) multi-stage Runge-Kutta method provided suitable boundary conditions. For hyperbolic problems, this type of time integrator is preferred because it maintains stability properties of the continuous equations such as total variation stability; hence, they prevent spurious energy build-up even in the presence of shocks (see for example (Gottlieb et al. 2011)). Due to superior stability properties demonstrated in (Kubatko et al. 2008), we used a three-stage, second-order Runge-Kutta method (RK23) for this study:

$$
\begin{aligned}
\mathbf{U}_{k}^{n+\frac{1}{3}} & =\mathbf{U}_{k}^{n}+\frac{\Delta t}{2} \mathbf{H}\left(\mathbf{U}_{k}^{n}\right) \\
\mathbf{U}_{k}^{n+\frac{2}{3}} & =\mathbf{U}_{k}^{n+\frac{1}{3}}+\frac{\Delta t}{2} \mathbf{H}\left(\mathbf{U}_{k}^{n+\frac{1}{3}}\right) \\
\mathbf{U}_{k}^{n+1} & =\frac{1}{3}\left(\mathbf{U}_{k}^{n}+2 \mathbf{U}_{k}^{n+\frac{2}{3}}\right)+\frac{\Delta t}{3} \mathbf{H}\left(\mathbf{U}_{k}^{n+\frac{2}{3}}\right),
\end{aligned}
$$

where a slope limiter is applied to the prognostic variables after every intermediate step. The slope limiter we are using is velocity-based and non-destructive with respect to wellbalancing and non-negativity preservation. It modifies $(h$, $h \mathbf{u})^{\top}$ in elements where artificial gradients occur and sets $h=0$ for small water depth $h<\epsilon_{\mathrm{tol}} \mathrm{m}$, provided a userdefined and test case-dependent parameter $\epsilon_{\mathrm{tol}}$. This will remove oscillations within elements that are typically treated by using dynamic viscosity as in (Marras et al. 2016); hence, an additional viscosity source term is not needed for the presented test cases. More detail can be found in (Vater et al. 2019) where the limiter was successfully applied to tsunami benchmark test cases and to model flood scenarios. In the present study, we show that it can be used as well for accurate shore line modelling and the reduction of spurious oscillations on adaptive meshes even when a variety of source terms are involved.

Hyperbolic problems are time step restricted and numerical stability of methods for their explicit solution depends on the Courant-Friedrich-Levy (CFL) condition (see Courant et al. 1928). Using a maximum possible value for a stable CFL number-we found $\mathrm{cfl}_{\max }=0.3$ a good choice for linear DG elements - and using the three-stage
Runge-Kutta method described above, we can compute a stable time step by

$\Delta t=\frac{\mathrm{cfl}_{\max } \cdot \Delta x^{\min }}{c^{\max }}$,

where $c^{\max }=\max _{k}\left(\left\|\mathbf{u}_{k}\right\|_{2}+\sqrt{g h_{k}}\right)$ is the global maximum shallow water velocity, with $k$ running over all degrees of freedom. The quantity $\Delta x^{\mathrm{min}}=\min _{k} \Delta x_{k}^{\mathrm{min}}$ is an estimate of the shortest height $\Delta x_{k}^{\mathrm{min}}$ of a triangle that is computed using the volume formula for triangles at the current time step leading to a global time step $\Delta t$. This is done before each time step allowing for a temporally changing and maximum possible global time step.

\subsection{Implementation of source terms}

Coastal flooding is a problem involving the interplay between many source terms. The source terms directly impact the momentum and thus the free surface. For reasons of comprehensiveness, we will give detail of their implementation in the following subsections.

\subsubsection{Earth's rotation}

Hurricanes can cover large areas up to $100 \mathrm{~s}$ or $1000 \mathrm{~s}$ of $\mathrm{km}^{2}$. On large geographic scale, Earth's rotation has a nonnegligible influence on water circulation. In the presented model, this Coriolis forcing $\tau_{C}$ is of the form $\tau_{C}=f(-h v$, $h u)^{\top}$ and $f=2 \omega \sin (\varphi)$ with $\varphi$ the latitude and $\omega=7.2921$. $10^{-5} \mathrm{rad} \mathrm{s}^{-1}$ the rotation rate of the Earth. A common approximation of the Coriolis force is the $\beta$-plane approximation, i.e. we linearly approximate $f=f_{0}+\beta y$, where $f_{0} \in \mathbb{R}, y$ is a planar coordinate and $\beta \approx \frac{2 \omega}{r_{E}}$ is a constant that depends on the Earth's rotation and radius $r_{E}$. We will use both parameterisations, the conventional and the $\beta$-plane approximation in this study.

\subsubsection{Bottom friction}

The bottom friction $\tau_{B}$ is assumed to take on the form of a quadratic Manning law $\boldsymbol{\tau}_{B}=\frac{g n^{2}\|\mathbf{u}\|_{2}}{h^{7 / 3}} h \mathbf{u}$, where $n$ is a dimensionless roughness parameter that is directly related to the nature of the bed and will take on values between 0.001 and 0.01 depending on the specifics of the test case. The bottom friction depends on the prognostic variables $\mathbf{U}$. In coastal areas, numerical flow directions might be reversed during wave run up due to small fluid depths and a resulting large friction term. To ensure stability of the numerical result, we follow the split-implicit time stepping procedure in (Kesserwani and Liang 2012) to ensure that flow directions 
do not reverse within one time step. This means that friction terms for $h<\varepsilon_{\text {tol }} \mathrm{m}$ and $\|h \mathbf{u}\|_{2}<\varepsilon_{\text {tol2 }} \mathrm{m}^{2} \mathrm{~s}^{-1}$ are neglected, where $\varepsilon_{\text {tol }}$ is the wet/dry tolerance of the slope limiter mentioned in Sect. 2 and $\varepsilon_{\text {tol } 2}$ is another small parameter. We chose $\varepsilon_{\text {tol }} \in\left[10^{-4}, 10^{-2}\right]$, and $\varepsilon_{\text {tol } 2}=10^{-8}$ throughout this study. In the first momentum equation for $h u$, we then compute for every degree of freedom

$$
\begin{gathered}
\mathrm{Sf}=-g^{4 / 3} n^{2} \frac{h u\|h \mathbf{u}\|_{2}^{2}}{h^{7 / 3}} \\
D=1-\Delta \operatorname{tg}^{4 / 3} n^{2} \frac{(2 h u)^{2}+(h v)^{2}}{h^{7 / 3}}\|h \mathbf{u}\|_{2}^{2}
\end{gathered}
$$

Then, if $\Delta t \mathrm{Sf} / D<|h u|$, the flow will not reverse within that time step and we can compute the friction term as $\Delta t \mathrm{Sf} / D$; otherwise, the friction is set to 0 .

Analogously, the bottom friction can be computed for the second momentum equation by interchanging the roles of $u$ and $v$.

\subsubsection{Bathymetry and well-balancing}

Non-linear interaction with bathymetry plays a crucial role in wave amplification. Special care has to be taken to prevent spurious waves caused by erroneous numerical approximations of bathymetry gradients. In this study, we solve the strong form (Eq. (3)) of the equations, which plays a crucial role in the following discussion. Well-balancing, i.e. preservation of the steady state at rest, is a desirable property of numerical discretisations. The steady state at rest assumes bathymetry gradients to be the only present source term. While well-balancing is achieved by setting gravity to 0 in cells adjacent to the wet/dry interface (Vater et al. 2019), the strong form is well-balanced on a discrete level without any modification when the flux divergence $\nabla \cdot \mathbf{F}(\mathbf{U})$ is discretised after differentiation, since in general

$\nabla \cdot\left(\sum_{k}\left(\mathbf{F}\left(\mathbf{U}_{k}\right)\right) \phi_{k}(\mathbf{x})\right) \neq \sum_{k} \mathbf{F}_{d i v}\left(\mathbf{U}_{k}\right) \phi_{k}(\mathbf{x})$.

where $\mathbf{F}_{d i v}\left(\mathbf{U}_{k}\right)$ and $\mathbf{F}\left(\mathbf{U}_{k}\right)$ are the nodal coefficients of the divergence of the flux and the flux, respectively. Since edgebased terms are always balanced, we show that for every wet element $\Omega_{i}$ we obtain

$\mathbf{F}_{\text {div }}\left(\mathbf{U}_{k}\right)=S\left(\mathbf{U}_{k}\right)$.

for $\mathbf{u}=\mathbf{0}$. Equation (7) is fulfilled as long as the flux divergence and the source evaluated at the Lagrange points are balanced. This will always be the case in fully wet cells for the water at rest where $\mathbf{u}=\mathbf{0}$, as

$\mathbf{F}_{d i v}\left(\mathbf{U}_{k}\right)=\left(0, h_{k} \nabla h_{k}\right)^{\top}=\left(0,-h_{k} \nabla b_{k}\right)^{\top}=S\left(\mathbf{U}_{k}\right)$,

always holds true for all degrees of freedom $k$. This balance is not achieved if the order of differentiation and discretisation of $\nabla \cdot \mathbf{F}(\mathbf{U})$ are reversed. This can be easily shown by a counter example on a master element with edges $(-1,-1),(-1,1),(1$, $-1)$ and using linear Lagrange polynomials.

\subsubsection{Wind fields and wind drag coefficients}

The wind stress is $\tau_{W}=\gamma_{\tau} \boldsymbol{\tau}$ with a wind friction $\gamma_{\tau} \in \mathbb{R}_{+}$that models the energy transfer from the atmosphere to the ocean. Given an external wind field $\mathbf{v}$, the wind stress can be computed as

$\boldsymbol{\tau}(\mathbf{v})=c_{d} \rho_{a}\|\mathbf{v}\|_{2} \mathbf{v}(\mathbf{x}, t)$

where $c_{d} \in \mathbb{R}$ is a drag coefficient and $\rho_{a}$ is the air density which we assume to be $1.15 \mathrm{~kg} \mathrm{~m}^{-3}$. The dimensionless drag coefficient $c_{d}=c_{d}(\mathbf{v})$ depends on wind speeds. For hurricane models, several different wind drag parameterisations have been explored and commonly used ones are depicted in Fig. 2 (left). In Garrat (1977) (blue line), observations from the past 10 years are used to show that for absolute wind speeds $|\mathbf{v}|$ within a range of 4 to $21 \mathrm{~m} \mathrm{~s}^{-1}$ the drag coefficient fulfils a linear relationship $c_{d} \cdot 10^{3}=0.75+0.067|\mathbf{v}|$ or a power law of the form $0.51|\mathbf{v}|^{0.46}$. We note that the understanding is that the drag coefficient is dimensionless and units are removed from occurring velocities $|\mathbf{v}|$. In Weisberg and Zheng (2006) (gray line), a drag coefficient as in (Large and Pond 1981) is used to study sensitivities with respect to approach speed, direction of approach and landfall location (see also Sect. 4.1 of this manuscript). Finally, Powell (2007) discusses wind drags for more extreme winds $|\mathbf{v}|>50 \mathrm{~m} \mathrm{~s}^{-1}$ and numerically computes drag coefficients that exceed those known in the literature. Their new drag coefficients show improved results for practical applications.

\subsubsection{Atmospheric pressure}

The atmospheric pressure gradient $\nabla p_{A}$ models part of the influence of the atmosphere onto the water column. In areas of relatively low air pressure in comparison to the ambient air pressure, this causes the water surface to slightly bulge upwards, increasing $h$ in this area. The influence of the pressure gradient is non-negligible: Numerical observations show that about $10 \%$ of the resulting surge can be attributed to the pressure gradient. It is implemented in a straight forward manner, utilising the local derivatives of the Lagrange polynomial expansion. The contribution of the gradient of the atmospheric pressure to the source integral can be written as

$$
\begin{aligned}
& \frac{\partial p_{A}}{\partial x}\left(\boldsymbol{\xi}_{k}\right)=\sum_{l}\left(\left.\sum_{m} \frac{\partial \phi_{l}}{\partial x}\right|_{\boldsymbol{x}_{m}} \phi_{m}\left(\boldsymbol{\xi}_{k}\right)\right) p_{A}\left(\mathbf{x}_{l}\right) \\
& \frac{\partial p_{A}}{\partial y}\left(\boldsymbol{\xi}_{k}\right)=\sum_{l}\left(\left.\sum_{m} \frac{\partial \phi_{l}}{\partial y}\right|_{\boldsymbol{x}_{m}} \phi_{m}\left(\boldsymbol{\xi}_{k}\right)\right) p_{A}\left(\mathbf{x}_{l}\right)
\end{aligned}
$$



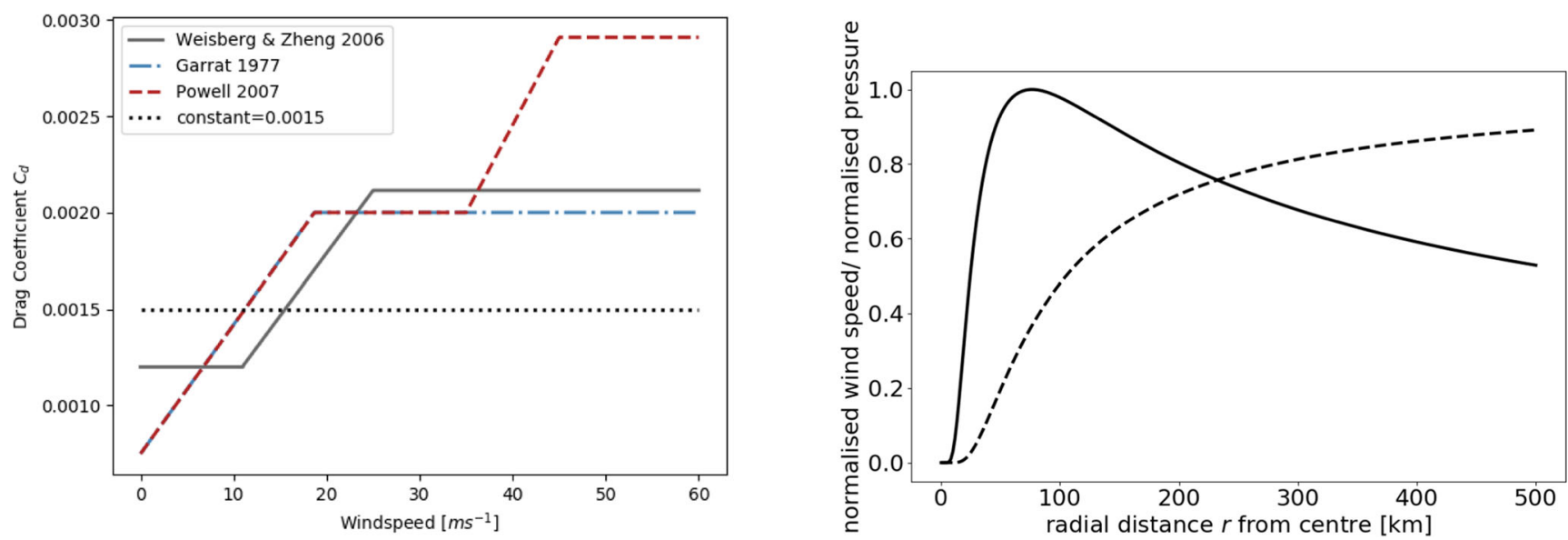

Fig. 2 Wind drag coefficients $c_{d}$ for hurricane storm surge models (left), Normalised wind speed (solid line) and pressure (dashed line) profiles using Holland's model (right). Wind speeds are normalised by $v_{\max }=\max _{\|x\|_{2}} v$ and pressures by the difference between ambient and central pressure $p_{n}-p_{c}$

where $\boldsymbol{\xi}_{k}$ are the Gauss-Legendre quadrature points, $\boldsymbol{x}_{l}$ are Lagrange interpolation points and the matrix multiplication inside the parentheses projects the derivatives of the basis functions $\phi_{l}$ onto quadrature points $\boldsymbol{\xi}_{k}$. Note that the model projects every triangle onto a master triangle, so that computationally, these matrix entries are constants and the matrix multiplication only has to be done once.

\subsection{Adaptive mesh refinement}

The computational model as described at the beginning of this Sect. 2 uses the mesh generator amatos (Behrens et al. 2005) to create dynamically adaptive and conforming triangular meshes. Smaller triangles are obtained by bisection (Rivara 1984). While the numerical method works equally well on truly unstructured meshes (as demonstrated in (Vater et al. 2019)), the more structured hierarchical mesh refinement approach has a number of computational advantages that are of interest here. A depth-first traversal of the refinement tree gives rise to a space filling curve that allows for optimisation of the memory layout as well as computational organisation for optimised parallel computations (see, e.g. Behrens 2005; Behrens and Bader 2009).

The dynamic grid manipulation involves problemdependent refinement indicators $\eta_{\Omega_{i}}$ for each element $\Omega_{i}$ (Behrens 2006) to control the element-wise refinement or coarsening. A commonly used example for a useful indicator is the gradient of the total water height at time $t$ :

$\eta_{\Omega_{i}}(t)=\max _{x \in \Omega_{i}}\|\nabla \boldsymbol{H}(\mathbf{x}, t)\|_{2}$.

For applications such as idealised hurricane storm surge, see also Sect. 4.1 of this manuscript, a composite refinement indicator might be necessary that refines areas of bathymetry gradients as well as areas of strong winds. The indicator we used for this purpose is defined as
$\eta_{\Omega_{i}}(t)=|\nabla b(\mathbf{x})|_{\Omega_{i}, 1}+|\mathbf{u}(t)|_{\Omega_{i, 2}}$

with $\mathbf{u}=(u, v)^{\top}$ the horizontal velocity vector and $|\cdot|_{\Omega_{i}, 1},|\cdot|_{\Omega_{i}, 2}$ the discrete $L_{1}$, and $L_{2}$ norm on element $\Omega_{i}$ respectively.

The width of elements is controlled by user-defined mesh levels $\lambda_{\text {ref }}$ and $\lambda_{\text {crs }}$ with $\lambda_{\text {crs }} \leq \lambda_{\text {ref. }}$. Starting from a macrotriangulation, the mesh is uniformly refined $\lambda_{\text {crs }}$ times until the coarse mesh level is reached. Subsequently, the refinement indicator is computed to determine elements that need to be flagged for refinement or coarsening. Moreover, user-defined tolerances $0 \leq \theta_{\text {crs }}<\theta_{\text {ref }} \leq 1$ determine the fraction of the domain to be modified as follows:

$\begin{array}{ll}\text { if } \quad \eta_{\Omega_{i}} \leq \theta_{\text {crr }} \eta_{\max } & \rightarrow \text { coarsen element } \Omega_{i}(11) \\ \text { if } \quad \eta_{\Omega_{i}} \geq \theta_{\text {ref }} \eta_{\max } & \rightarrow \text { refine element } \Omega_{i}\end{array}$

with $\eta_{\max }=\eta_{\max }(t)=\max _{\Omega_{i} \subset \Omega} \eta_{\Omega_{i}}(t)$ the maximum value of the refinement indicator over all elements at time $t$. This manipulation strategy is carried out in a loop until the desired finest mesh level $\lambda_{\text {ref }}$ is reached. This approach will produce meshes that consist of small elements on mesh level $\lambda_{\text {ref }}$ in the area of interest with a continuous but narrow transition zone to coarse elements on mesh level $\lambda_{\text {crs }}$ outside the area of interest.

The node values are then interpolated or restricted after modification using the known Lagrange basis functions for each element. In regions close to the waterline, we keep the mesh relatively fine (see also Sect. 4.1) to avoid well-balancing issues. The refinement process has the further advantage of using a cache-efficient space-filling curve-ordering of elements (Behrens and Bader 2009), which allows fast access of neighbouring elements: This feature is particularly beneficial for local numerical discretisations such as discontinuous Galerkin method since elements only communicate over edges.

For convenience, the meshes are kept conforming, i.e. free of hanging nodes, throughout the simulation. We stress that 

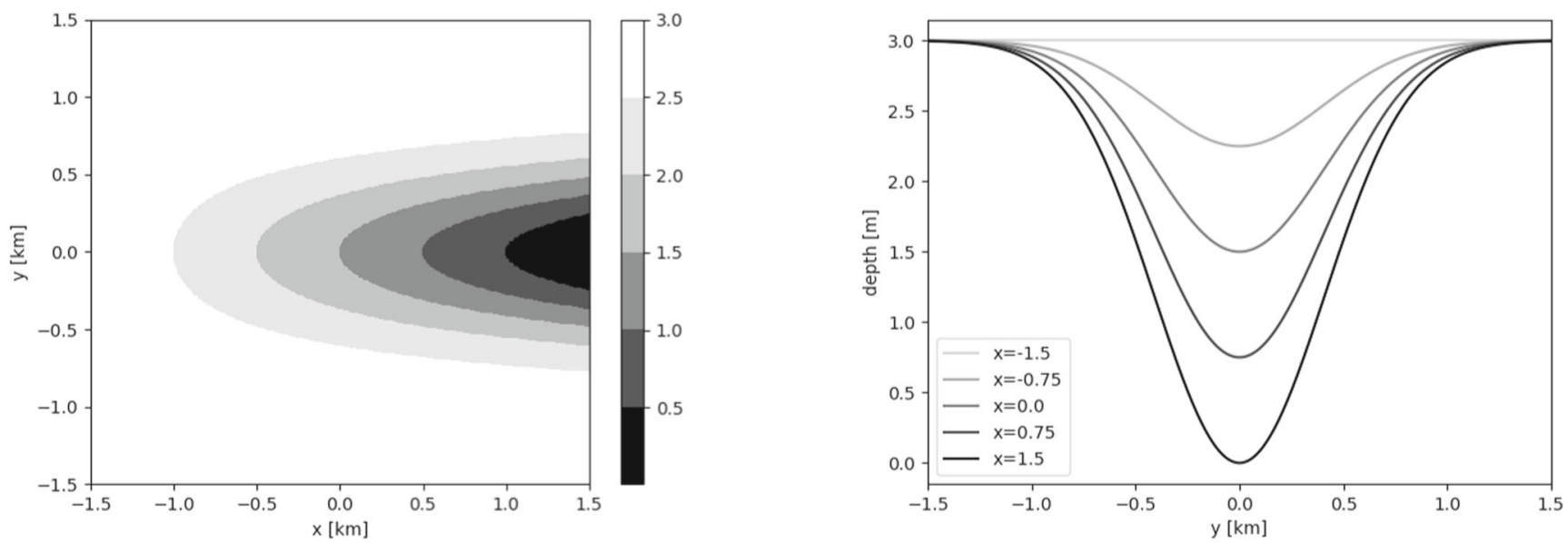

Fig. 3 Hypothetical embayment: 2D plot of bathymetry profile (left) and cross sections (right)

this is not required by the method itself. Hanging nodes would require to combine two or more Riemann solutions over one (coarse) edge as was recently demonstrated (Hermann et al. 2011; Kopera and Giraldo 2014).

\section{Results I: Source terms}

In this section, we present test cases to show the major functionalities of the model. Note that physical dimensions are not explicitly given, but we assume throughout this paper that we are using SI base units. In some cases, we chose to not use SI base units to increase readability. In those cases, the dimensions are explicitly mentioned. The following subsections will in particular focus on the aspects of:

- Inundation stability and conservation properties (Sect. 3.1)

- Balanced wind and pressure forcing (Sect. 3.2)

- Robustness of wind forcing (Sect. 3.3)

\subsection{Tidal flow in a symmetric embayment}

Here, we show one configuration of the simulation of an embayment first presented in Ip et al. (1998). Similar simulations have been reported on in Luettich and Westerink (1995) using the finite element model ADCIRC as well as in Dawson et al. (2011) using another discontinuous Galerkin model and a modified bathymetry. This simulation is to show idealised estuarine flooding by an in- and outgoing tide to show inundation stability and conservation properties of the model.

On a square domain $\Omega=[-L, L]^{2}$ with $L=1500$, a symmetric bathymetry as shown in Fig. 3 is defined by $b(\mathbf{x})=$ $3.0-(1.50+\widehat{x}) \mathrm{e}^{-3 \hat{y}^{2}}$, where we use the definition $\widehat{x}=10^{-3} x, \widehat{y}$ $=10^{-3} y$. Moreover, the water surface is assumed to be in a steady state at rest. On the right boundary of the domain (at $x=L$ ), a tidal forcing is prescribed as

$\mathrm{H}(\boldsymbol{x}, t)=h_{\mathrm{de}}+3 \sin \left(\frac{2 \pi t}{T}\right)$,
Fig. 4 Hypothetical embayment: cross sections of the solution for $\mathrm{x} \in[-1500,-1000]$ projected onto $\mathrm{y}=500$ at times $\mathrm{t}=0,31.1$, and 62.1 hours (top to bottom)
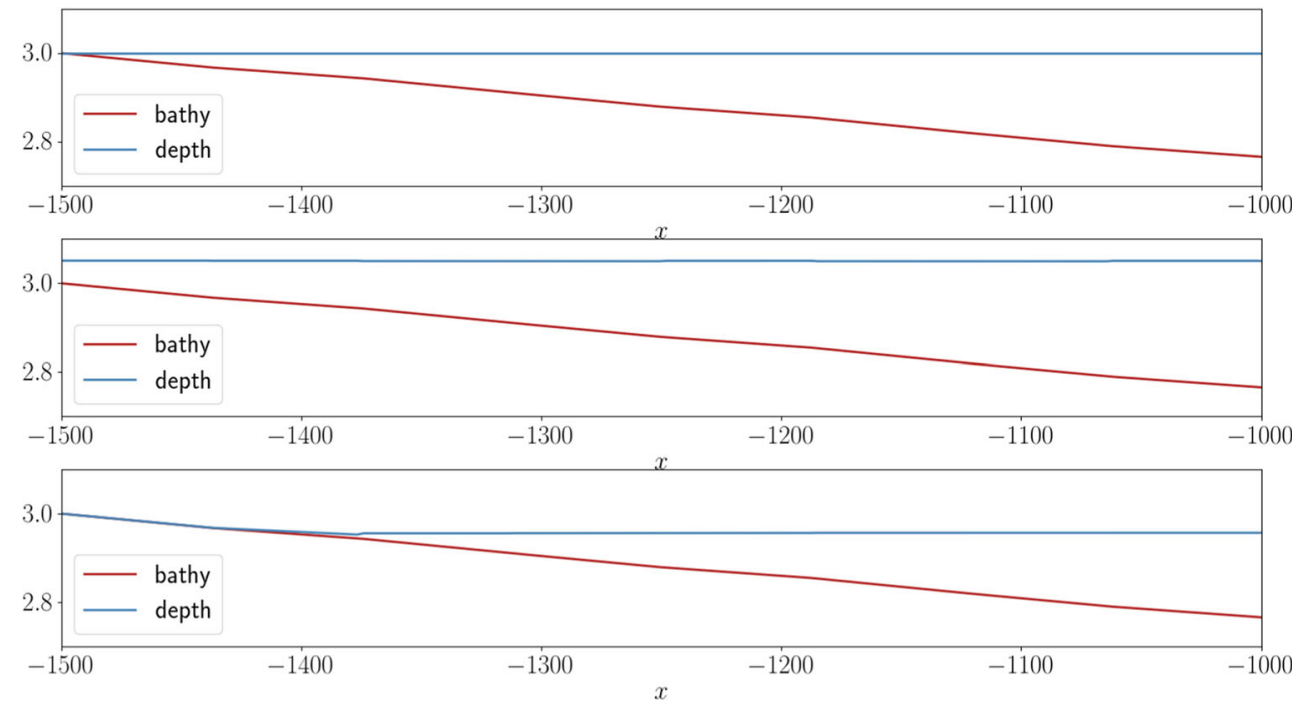

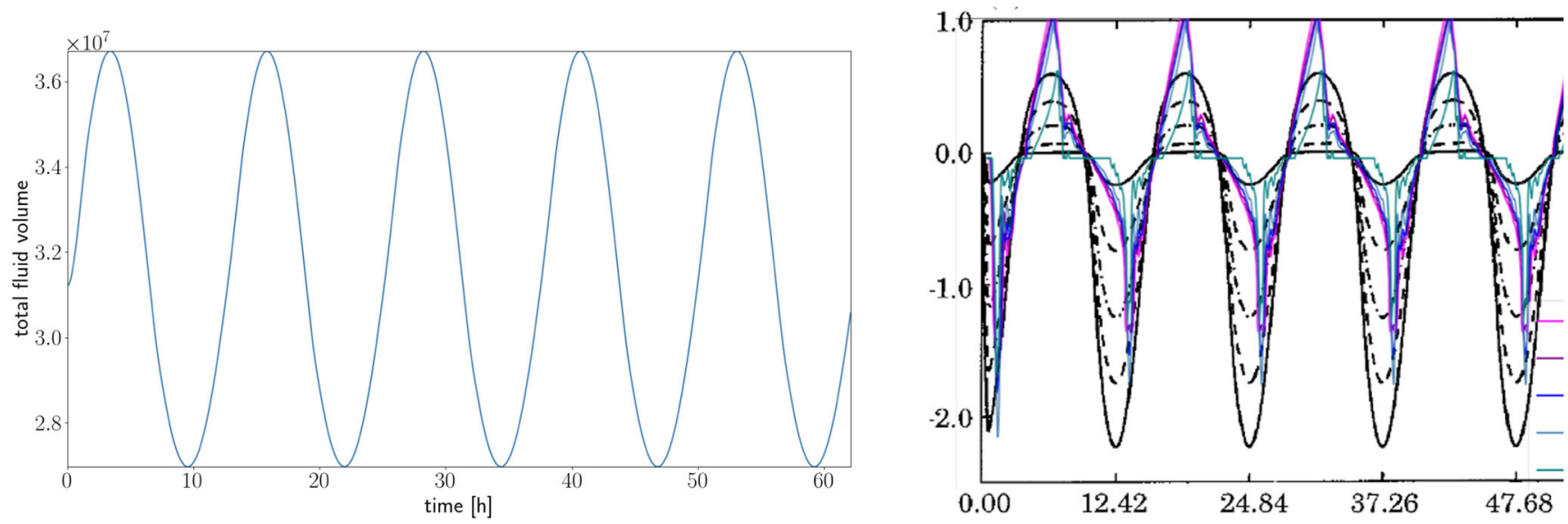

Fig. 5 Hypothetical embayment: plot of fluid volume (left) and comparison of integrated flux $H u$ (coloured lines) with the results presented in Ip et al. (1998) (black lines) for four full tidal cycles over cross sections at $\hat{x} \in\{0, \pm 0.6, \pm 1.2\}]$ (right) over time

for $x=L, y \in[-L, L]$, where $T$ is the $M_{2}$ tide, i.e. a temporal period of $T=4,471,200$ (equivalent to 12.42 hours) is prescribed (Ip et al. 1998) and $h_{\mathrm{de}}$ denotes the water depth in the deepest end of the domain. All other boundaries are set to be impermeable. Friction is assumed to be negligible, i.e. $n=0$. For the initial water surface which is at rest, the water surface is levelled with the highest point of the bathymetry $b_{\max }=3.0$.

To avoid polluted results caused by the definition of the boundary condition, we artificially increased the domain by $L$ to the right, i.e. for $\widehat{x} \geq 1.5$, and assume the bathymetry in this area to be identically 0 , hence adding a discontinuity to the bathymetry. As we will see, this did not pose a problem to our robust numerical method. We ran five full tidal cycles with a time step of $\Delta t \approx 4$, a spatial resolution of $\Delta x=93.75$ and a CFL number of 0.3 which we found to be the largest possible CFL number for this particular test case that allowed for a stable computation and which is similar to the maximal CFL numbers found in (Xu et al. 2014) for similar applications. The determination of theoretical bounds for CFL numbers for multi-dimensional problems is difficult, and most results are restricted to one space dimension (Kubatko et al. 2014). Snapshots of the numerical solutions over a cross section at $y=500$ are found in Fig. 4 which shows that wetting and drying is occurring on the left boundary of the domain and, hence, that the inundation scheme is stable. We observe- see left display in Fig. 5-global mass conservation in agreement

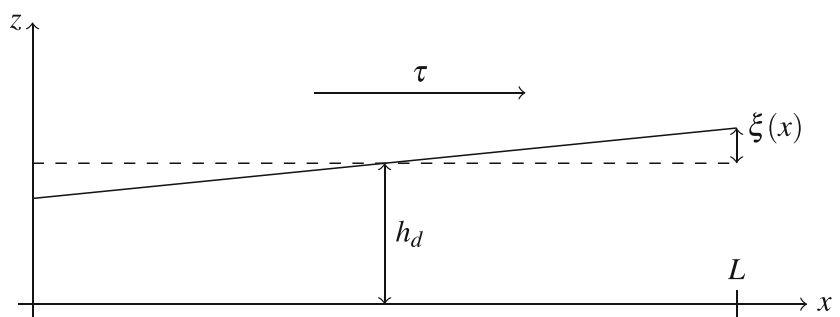

Fig. 6 Steady-State Wind Test Case: 1D projection of the initial conditions. In this figure, $z$ denotes a vertical coordinate with (Ip et al. 1998) showing that the limiter presented in (Vater et al. 2019) is non-destructive with respect to global mass conservation and that the variation of total fluid volume due to the tidal in/outlet is of the order of $\mathcal{O}\left(10^{7}\right)$. We note that the model used in (Ip et al. 1998) achieves a much larger time step of $\Delta t=111.78$ at a spatial resolution of $\Delta x=75$. We attribute this to the fully implicit evolution scheme that their model uses.

Furthermore, we numerically computed the integral of the flux $H u$ over cross sections at $x \in\{ \pm 1200, \pm 600,0\}$ :

$\int_{-L}^{L}(h+b)(x, y) \cdot u(x, y) d y$

The results are shown in the right display of Fig. 5. We see that after a short calibration time, the results match the results in (Ip et al. 1998) well and yield values for the cumulative transport of the order $10^{3}$. We note that especially at $\widehat{x}=-1.20$, we still get meaningful and non-zero results. This is notable because that part of the domain is close to the left hand boundary and therewith exposed to wetting and drying (see Fig. 4 for a close-up) and shows that the slope limiting strategy is gentle enough to reduce spurious oscillations while also yielding realistic values for velocities. Finally, we point out that the results are found to not be sensitive to the parameter $\epsilon_{\mathrm{tol}}$ that determines a cut-off value for small water depths. We ran the simulation with $10^{-6} \leq \epsilon_{\text {tol }} \leq 10^{-3}$ and did not find different results.

Table 1 Steady-State Wind Test Case: analytical solution $h_{\text {ana }}$, and numerical solution $h$ at discrete points $x \in\{500,10,500,20,500\}$

\begin{tabular}{llll}
\hline$x \mathrm{~m}$ & 500.00 & $10,500.00$ & $20,500.00$ \\
Analytical solution $\xi_{\text {ana }}(x)$ & $-2.04 \cdot 10^{-2}$ & $0.00 \cdot 10^{-2}$ & $2.04 \cdot 10^{-2}$ \\
$\quad h_{\text {ana }}(x)=h_{d}+\xi_{\text {ana }}(x)$ & 4.9706 & 5.0000 & 5.0204 \\
Numerical solution $\xi(x)$ & $-2.04 \cdot 10^{-2}$ & $0.00 \cdot 10^{-2}$ & $2.04 \cdot 10^{-2}$ \\
$\quad h(x)=h_{d}+\xi(x)$ & 4.9706 & 5.0000 & 5.0204 \\
\hline
\end{tabular}


Fig. 7 Steady-State Wind Test Case: relative error at $x=500$ (solid line), $x=10,500$ (dashed line), and $x=20,500$ (dashed dotted line)

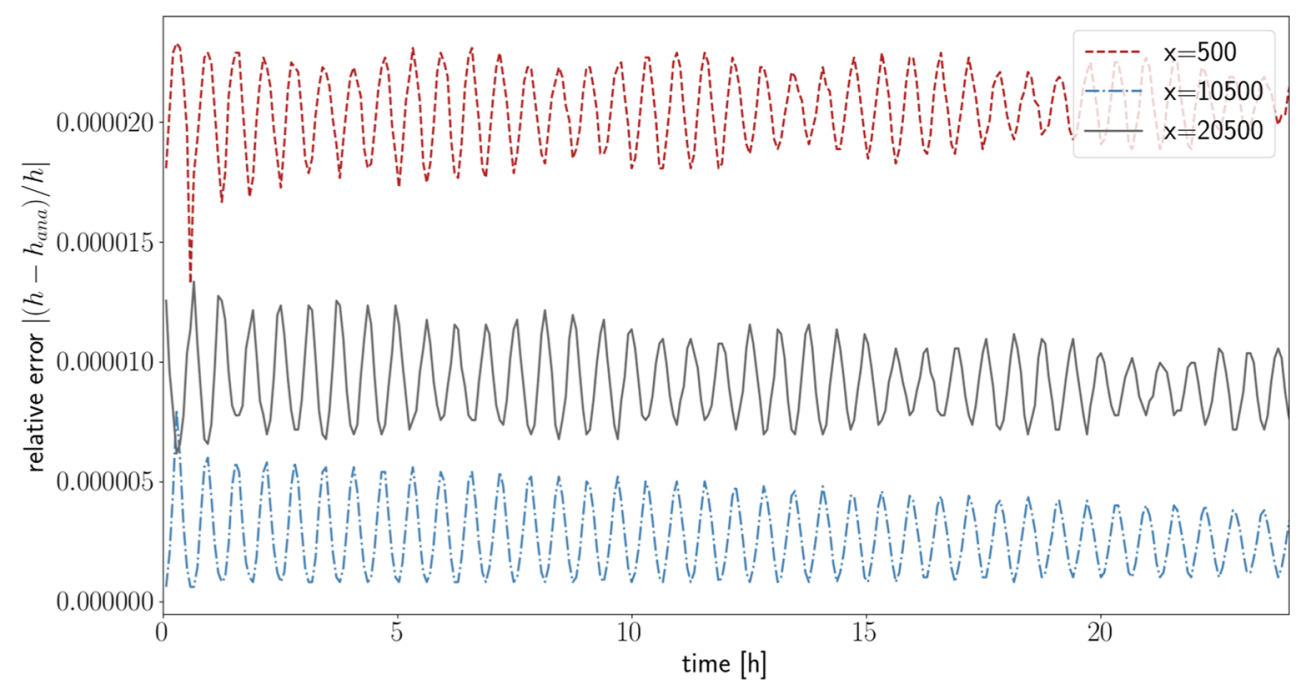

$g\left(h_{d}+\xi\right) \frac{\partial}{\partial x} \xi(\mathbf{x}) \stackrel{!}{=} \frac{1}{\rho} \tau_{1}(\mathbf{x})$,

This test case was described in (Davis and Sheng 2003). In a rectangular basin, $\Omega=[0, L] \times[0, D]$ with dimensions $L=$ 21000 and $D=5000$ with constant bathymetry $b(\mathbf{x})=0$ and impermeable boundaries on all four edges; a linear water surface $h_{d}+\xi$ is described as shown in Fig. 6. Here, $h_{d}=5$ and

$\xi(\mathbf{x})=\frac{\tau_{1}}{g h_{d} \rho}\left(x-\frac{L}{2}\right)$,

with $g$ the acceleration due to gravity, $\rho=10^{3} \mathrm{~kg} \mathrm{~m}^{-3}$, the water density, and absent initial velocities $\mathbf{u}=\mathbf{0}$. A constant wind stress is assumed as $\tau=\left(\tau_{1}, \tau_{2}\right)^{\top}=(0.1,0)^{\top}$ and $\gamma_{\tau}=1$ (for the definition of $\gamma_{\tau}$ see also 2.1.4). In previous studies (Davis and Sheng 2003; Qiang et al. 2016), the steady state is described as shown in Table 1. The main balance here is to be achieved in the $x$-momentum equation between the flux divergence $\nabla \cdot \mathbf{F}(\mathbf{U})$ and the wind stress, i.e. on a discrete level. Here we used the symbol $\stackrel{!}{=}$ to indicate that the balance is to be enforced. The partial derivative of $\xi$ can be computed as $\frac{\partial}{\partial x} \xi(\mathbf{x})=\tau_{1}\left(g h_{d} \rho\right)^{-1}$ as $\xi$ is a linear function of $x$. Substituting in the derivative of $\xi$ and $\tau_{1}=0.1$ in Eq. (10), we get after division by $g$ :

$\left(h_{d}+\xi\right) \cdot\left(\frac{0.1}{g h_{d} \rho}\right)=\frac{0.1}{g \rho} \Leftrightarrow\left(h_{d}+\xi\right)=h_{d}$.

This equality is approximately satisfied because $\xi \ll h_{d}$ by definition, i.e. $\frac{h_{d}+\xi}{h_{d}} \approx 1$.

For the simulation, we used a variable time step with a CFL number of 0.3 which is close to the theoretical maximum and was found to lead to stable results and a Runge-Kutta time stepping scheme of second order (RK23) until $T_{\text {end }}=10^{4}$ with
Fig. 8 Steady-State Wind Test Case: surface elevation (top) and meridional velocities (middle and bottom) at cross section $y=$ 2500 at times $t \in\left[0, T_{\text {end }} / 2, T_{\text {end }}\right]$ in colour and for all other times in between in light gray

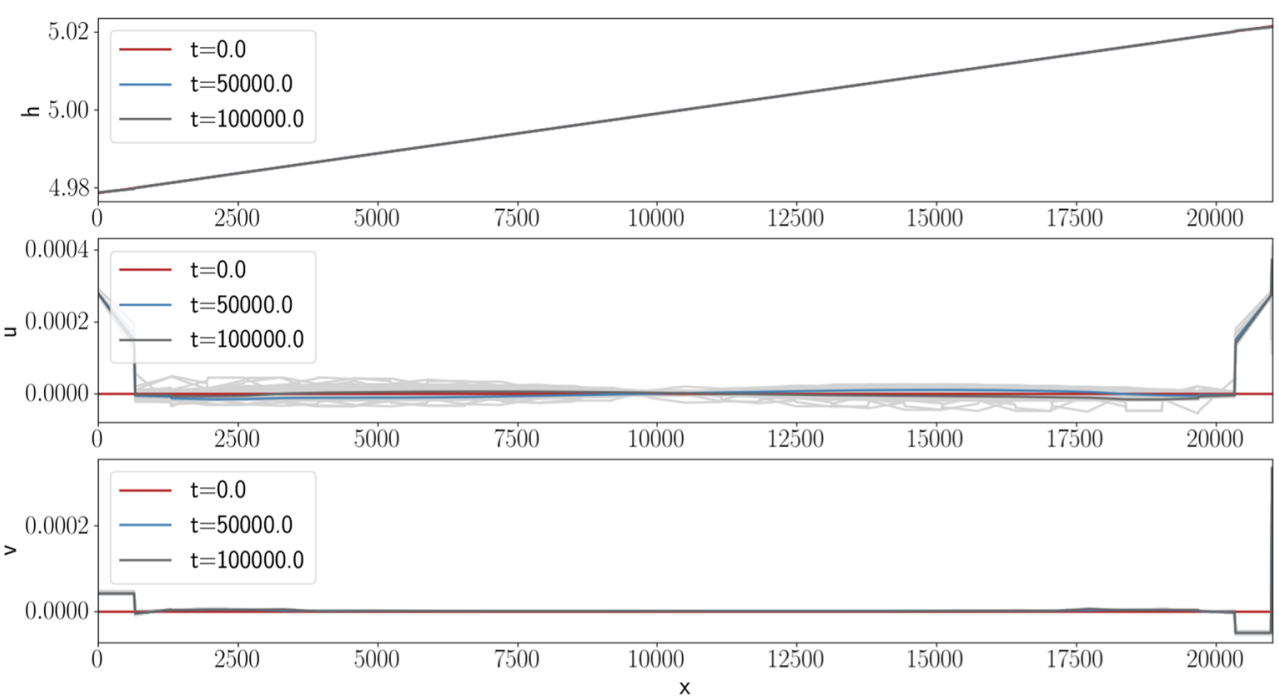


Fig. 9 Wind-induced circulation in a semi-enclosed homogeneous, rotating basin: sketch of initial condition. Depicted are the bathymetry and initial water surface (left) and the wind field (right)
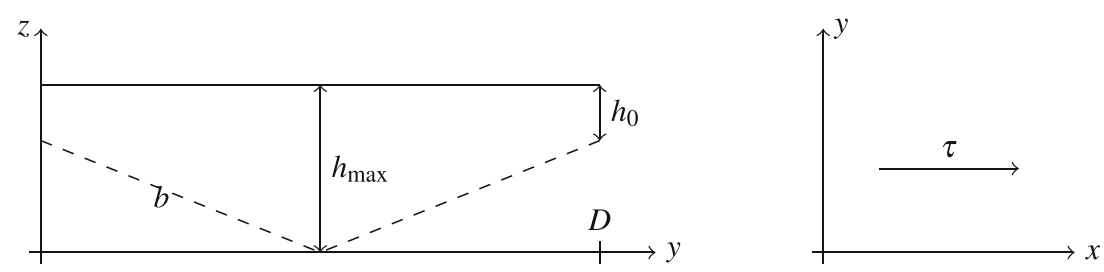

a spatial resolution of $\Delta x=656.3$. The Manning friction does not have a significant impact in this case as the numerical velocities are negligible. The dynamically computed time step using Eq. 7 was $\Delta t \approx 8.9$ throughout the simulation. The results are shown in Table 1 and Fig. 7. In Table 1, we compare point values at $t=T_{\text {end }}$ with the steady state solution $h_{\text {ana }}$ and find them to be identical. Figure 7 considers the relative errors in surface height $h$ at the numerical wave gauges at $x \in\{500,10,500,20,500\}$ over time in more detail. We observe that numerical errors are of the order $\mathcal{O}$ $\left(10^{-5}\right)$ and that they stay bounded over time. We remark that although possible in this DG method, we did not consider physical viscosity in these simulations. This is in contrast to the findings in (Davis and Sheng 2003) who solved RANS equations with horizontal and vertical eddy viscosities to achieve an exact solution. The reason for this is that their model allowed the definition of an eddy viscosity of the form $A_{H} \Delta \mathbf{u}$ and a boundary condition of the form $\partial_{z} A_{v} \partial_{z} \boldsymbol{u}$ at the free surface. By setting $A_{v} u_{z}=\tau / \rho$, they achieve an exact balance of the wind field and the gradient leading to an exact solution. Overall, we see that the steady-state values from Table 1 are matched and the wind and gravity forces are balanced. Our observed relative errors are of the order $\mathcal{O}\left(10^{-5}\right)$ and are decreasing with increasing spatial resolution. Figure 8 furthermore shows snapshots of the numerical solution over the cross section at $y=2500$ at discrete time steps. Shown are the fluid height and velocities for increments of 500 in light grey and for $t=0, T_{\text {end }} / 2, T_{\text {end }}$ in colour. We observe that due to the boundary conditions, small meridional velocities $\mathbf{u}=(u, v)^{\top}$ form at the walls at $(x=0$ and $x=21000)$. However, they stay bounded throughout the simulation, do not influence the surface height $h$ significantly and do not destroy the steadystate balance. We attribute this to enough numerical diffusion being present in the model to disperse energy and prevent build-up.

\subsection{Wind-induced circulation in a semi-enclosed homogeneous, rotating basin}

This test case is described in (Sanay and Valle-Levinson 2005). In a semi-enclosed rectangular domain $\Omega=[0$, $10 D] \times[0, D]$ with $D=10,000$, a piecewise linear bathymetry (see also the sketch in Fig. 9) of depth $h_{\max } \in \mathbb{R}$ is prescribed as follows: $b(\mathbf{x})=\frac{2\left(h_{\max }-h_{0}\right)}{D}\left|y-\frac{D}{2}\right|$.

Here, $\mathbf{x}=(x, y)^{\top}$ is the spatial coordinate and $h_{0}=3.0$ is the minimum water depth. The water surface is at rest at time $t=0$ and a constant wind stress $\tau=\left(\tau_{C}, 0\right)^{\top}, \tau_{C} \in \mathbb{R}$, aligned with the $x$ axis is prescribed and linearly ramped up over a period of 6 hours. Six different configurations of the parameters, which are given in Table 2, were tested to assess how the maximal occurring velocities are impacted by rotation, strength of the wind stress $\tau$, as well as depth of the basin $h_{\max }$.

We show simulation results on a uniform grid using 81,920 triangular linear elements with radii of the inscribed circles of about $\Delta r=38.5$. This corresponds to a spatial distance of $\Delta x=$ 781.25 and $\Delta y=156.25$ between grid points. On the left most edge of the basin, we used transmissive boundary conditions. Impermeable boundary conditions are prescribed on all other boundaries. A quadratic Manning law was used with $n=$ 0.0025 as was suggested in a previous study (Sanay and ValleLevinson 2005) to parameterise the bottom friction and a $\beta$-approximation to model the Coriolis forcing as described in Sect. 2.1.1 was used with $f_{0}=0.001$.

The results are found in Fig. 10. For all six configurations the magnitudes of the velocities are plotted in colour as well as the velocity vectors for every 100th point. Note that configurations 5 and 6 are scaled by factors of 10 and 0.2 respectively to improve readability.

Figure 11 shows cross sections of velocities (scaled with the maximum occurring velocity) at $x=50,000$ (mid basin) and $x=98,000$ (close to the head of the channel located at $x=100,000$ ). In general, we observe a re-circulation zone of below 10,000 from the head which is in line with the observations reported in

Table 2 Wind-induced circulation in a semi-enclosed homogeneous, rotating basin: parameters for all six experiments

\begin{tabular}{llll}
\hline Experiment number & $\tau_{C}$ in Pa & $f_{0}$ in s & $h_{\max }$ in m \\
\hline Configuration 1 & 0.080 & 0 & 20 \\
Configuration 2 & 0.080 & $10^{-4}$ & 20 \\
Configuration 3 & 0.080 & $10^{-4}$ & 60 \\
Configuration 4 & 0.080 & $10^{-4}$ & 8 \\
Configuration 5 & 0.008 & $10^{-4}$ & 20 \\
Configuration 6 & 0.500 & $10^{-4}$ & 20 \\
\hline
\end{tabular}



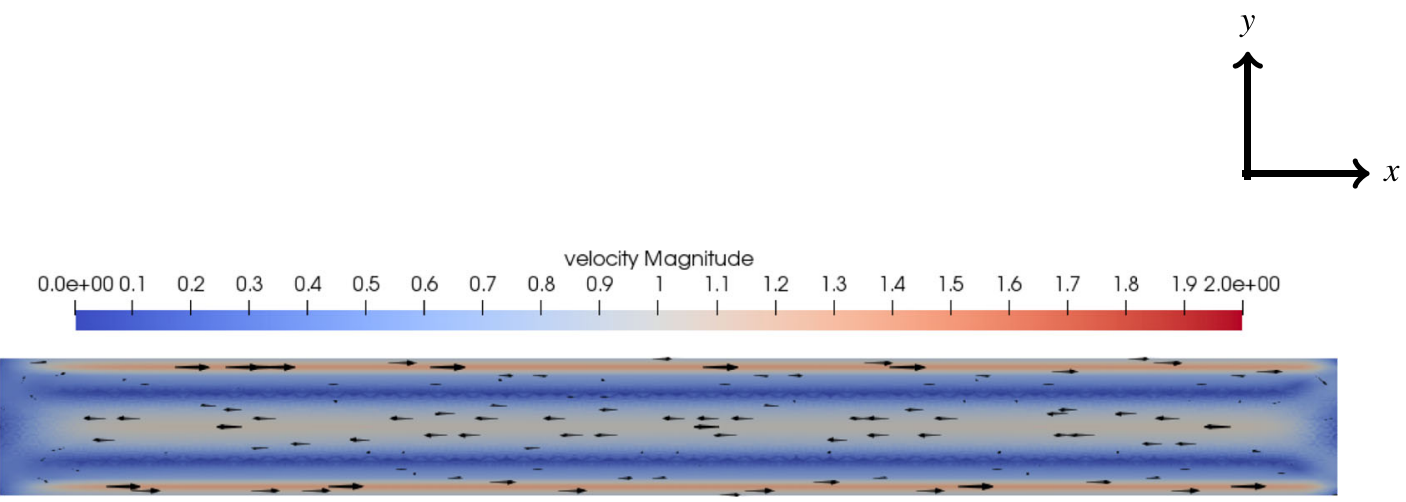

(a) Configuration 1

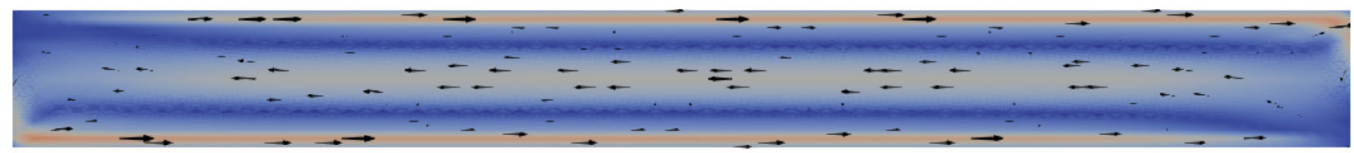

(b) Configuration 2

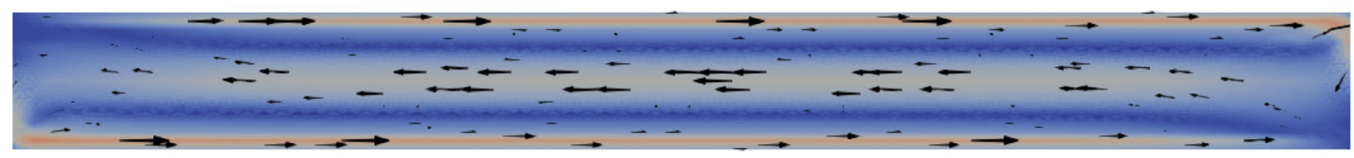

(c) Configuration 3

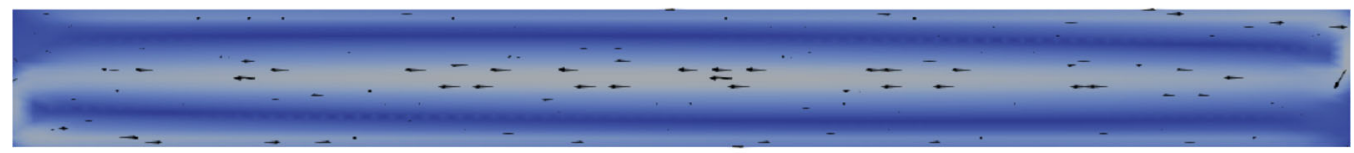

(d) Configuration 4

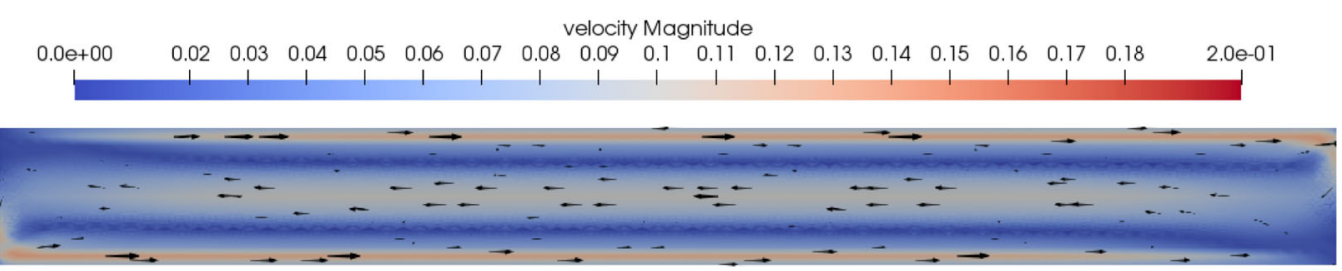

(e) Configuration 5

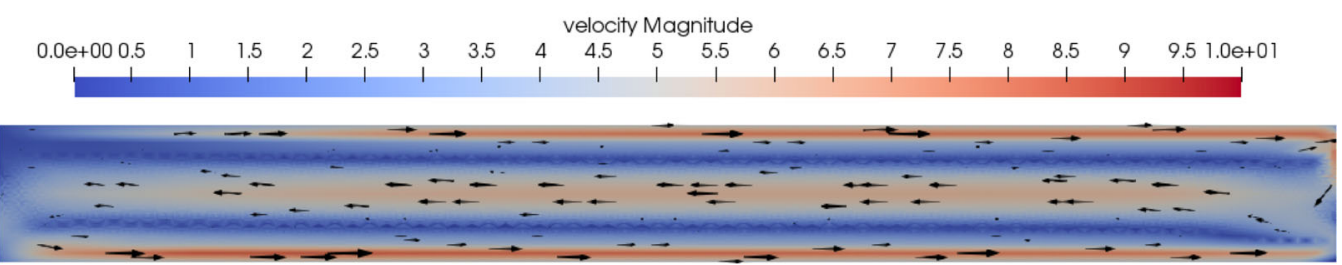

(f) Configuration 6

Fig. 10 Wind-induced circulation in a semi-enclosed homogeneous, rotating basin: velocities for all configurations (1-6) at time $t=37,500$ (top to bottom) 
(Sanay and Valle-Levinson 2005). The major characteristics of this test case are reproduced by the simulation results: We find a positive correlation between wind strength and velocities (two bottom panels of Fig. 10) as well as a negative correlation between water depth $h$ and the magnitude of the velocities $|\mathbf{u}|$. For the nonrotational case (configuration 1, top panel in Fig. 10), we agree with the observations from (Sanay and ValleLevinson 2005) and find a symmetric profile of the meridional velocities. This changes for all rotational test cases in which we find asymmetries near the head (at $x=98,000$ - see Fig. 11). We furthermore find (see Fig. 11) steeper gradients on the left shoal for configurations 2 and 3. For configuration 4, Sanay and Valle-Levinson (2005) find a symmetric velocity profile-almost as for configuration 1-this, we cannot confirm. However, we would like to point out that they used different equations to model this problem. Hence, an explanation might be found, again, in the characteristics of the shallow water equations. They are depth-integrated, so that the Coriolis force effects the entire water column. The model employed in (Sanay and Valle-Levinson 2005) used between 10 and 30 vertical layers in their model which we believe to add a dissipation that we are unable to reproduce.

Resolution appears to be a critical issue for this test case. With a spatial resolution of only $\Delta r=154$ (a total of 5120 triangles in $\Omega$ ), with $\Delta r$ is the radius of the inscribed circle, we observe that after long integration times instabilities develop in the form of vortices at the bottom end of the domain for all rotational configurations (2-6), indicating that the resolution is not sufficient for a realistic and physically correct solution. We attribute this effect to the depth-integrated character of the shallow water equations as well as the occurrence of a geostrophic imbalance. As opposed to the model used in (Sanay and Valle-Levinson 2005) that included several vertical layers and with that the ability to dissipate energy in the vertical dimension resulting in a rotation of the fluid in the $(y-z)$ plane, the depth integrated shallow water equations cannot take vertical motion into account.

\section{Results II: Adaptive mesh refinement}

In this section, we show and comment on the use of adaptive mesh refinement in the presented model. To do this, we will focus on two test cases:

- Idealised storm surge modelling and sensitivity analysis (Sect. 4.1)

- Idealised dam break (Sect. 4.2)

\subsection{Idealised hurricane approaching a linearly sloping coast}

In order to study the viability of the current model for use in hindcasts, we implemented an idealised test which is similar to a test presented in (Mandli 2011). It is designed to reproduce observations of a published
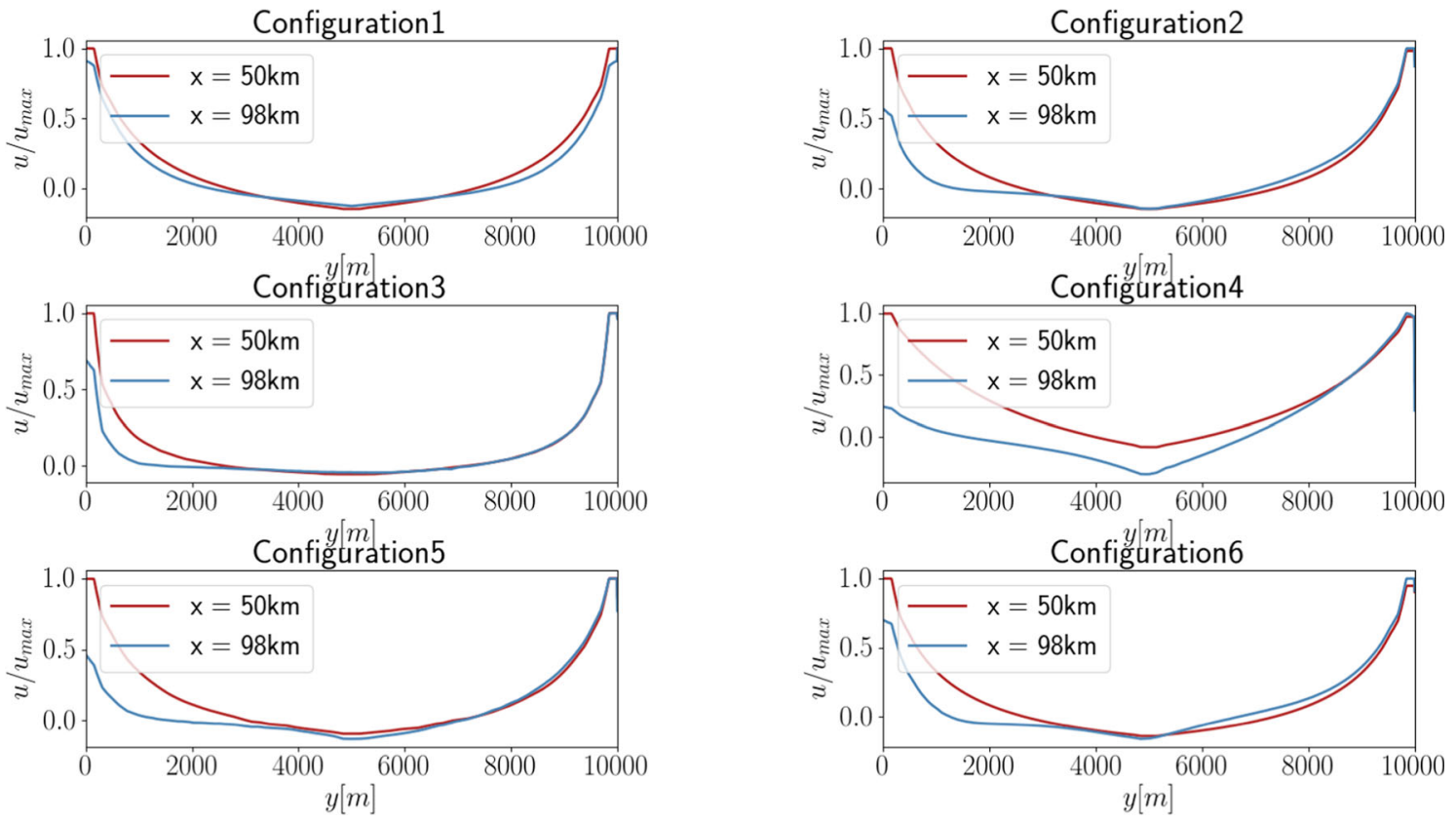

Fig. 11 Wind-induced circulation in a semi-enclosed homogeneous, rotating basin: scaled velocities at $t=20,000$ 


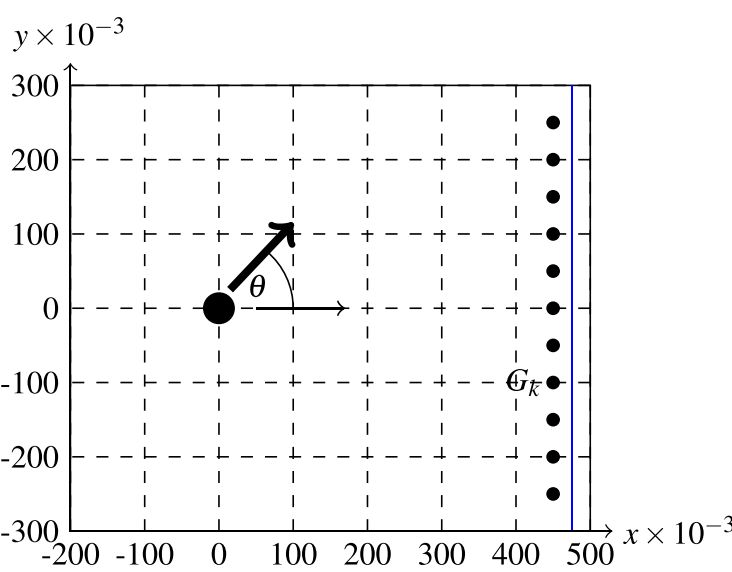

Fig. 12 Idealised hurricane approaching a linearly sloping coast: topdown view of set up with beach indicated by blue line, wave gauges by dots, the initial storm position by a large black dot, and the approaching

sensitivity study (Weisberg and Zheng 2006). The latter showed that hurricane flood intensity is sensitive to the storm's approach speed, direction of approach $\theta$ and landfall location. Our idealised test set up is defined as follows:

Let $\Omega=[-200,000,500,000] \times[-300,000,300,000]$ be a rectangular basin with a transmissive boundary at $x=-200,000$, impermeable boundaries otherwise and a bathymetry defined by the piece-wise linear function

$b(\mathbf{x})= \begin{cases}0 & \text { for } x \leq 350 \times 10^{3} \\ \alpha_{b} \cdot\left(x-350 \times 10^{3}\right) & \text { otherwise }\end{cases}$

where $\alpha_{b}=0.025$ is the slope of the bathymetry and $\mathbf{x}=(x, y)^{\top}$ is the spatial coordinate (see also Fig. 12). The initial water surface is at rest and described by $h(\mathbf{x}, 0)=\max (3000.0-b(\mathbf{x}), 0.0)$.

We then initialise a cyclone at an initial position (see large black dot in Fig. 12) and an approach angle $\theta$. The corresponding wind stress $\tau$ requires the computation of a continuous wind field. This can either be accomplished by using reanalysis data or a parameterised model that allows the derivation of a continuous wind field from a few discrete parameters; see (Beisiegel and Dias 2019) for a short discussion on using a

Table 3 Idealised hurricane approaching a linearly sloping coast: parameters for all six experiments

\begin{tabular}{llll}
\hline Configuration & Start point in km & $\begin{array}{l}\text { Approaching } \\
\text { angle, } \theta\left(^{\circ}\right)\end{array}$ & $\begin{array}{l}\text { Approaching } \\
\text { speed }\left(\mathrm{m} \mathrm{s}^{-1}\right)\end{array}$ \\
\hline 1 & $(0.0,0.0)^{\top}$ & 0 & 5 \\
2 & $(200.0,-100.0)^{\top}$ & -45 & 5 \\
3 & $(200.0,100.0)^{\top}$ & 45 & 5 \\
4 & $(425.0,-100.0)^{\top}$ & -90 & 5 \\
5 & $(425.0,100.0)^{\top}$ & 90 & 5 \\
6 & $(0.0,0.0)^{\top}$ & 0 & 25 \\
\hline
\end{tabular}

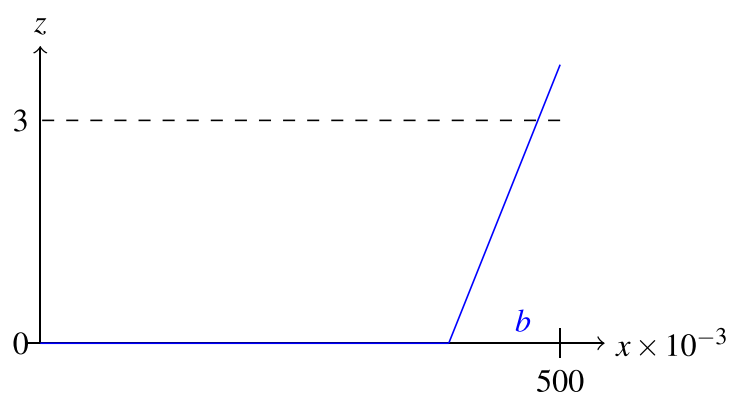

angle with $\theta$ (left); cross section of bathymetry (blue line) and resting water surface (dashed line) (right)

parameterised model in combination with re-analysis data for the Republic of Ireland. In the present model, the wind field is computed using a parameterised model (Holland 1980), which we elaborate on in more detail in Sect. 4.1.1, with parameters $p_{c}=95,000 \mathrm{~Pa}, p_{n}=100,500 \mathrm{~Pa}, A=23$ and $B=$ 1.5 which are representative for the 2008 hurricane Ike. Six different configurations as in (Mandli 2011) are implemented (cf. Table 3) and final times $T_{\text {end }}$ are chosen such that the storm's landfall is captured. Note, that we use oceanographic conventions, i.e. $0^{\circ}$ corresponds to travelling to the right. The boundary conditions are transmissive at $x=-200 \times 10^{3}$ and impermeable otherwise. Transmissive boundaries for this subcritical flow were implemented following (Antonopoulos and Dougalis 2016) using a standard approach based on Riemann invariants.

\subsubsection{Holland's model to compute hurricane winds}

The wind stress in Eq. (8) depends quadratically on the wind v. For hurricanes, winds can be computed using the model (Holland 1980):

$\mathbf{v}(\mathbf{x})=v(r) \cdot \mathbf{t} \quad$ with $\quad v(r)=\sqrt{\frac{A B\left(p_{n}-p_{c}\right) e^{-\frac{A}{r^{B}}}}{\rho_{a} \rho r^{B}}+\frac{r^{2} f^{2}}{4}}-\frac{r f}{2}$,

where $r$ is the distance from the centre of the storm; $\mathbf{t}$ the tangent to the circle with radius $r, A, B \in \mathbb{R}$ are shape parameters; $p_{n}$ and $p_{c}$ are the ambient and central pressure respectively; $\rho$ the water density; and $f$ the Coriolis parameter. The scaling parameters $A$ and $B$ are then obtained from the maximum wind speed as well as the radius of maximum winds (RMW):

$B=(\max |\mathbf{v}|)^{2} / \Delta p \cdot\left(\rho_{a} e\right)$ and $A=\mathrm{RMW}^{B}$,

where $e$ is Euler's number. An example for a normalised wind profile is found in Fig. 2. 


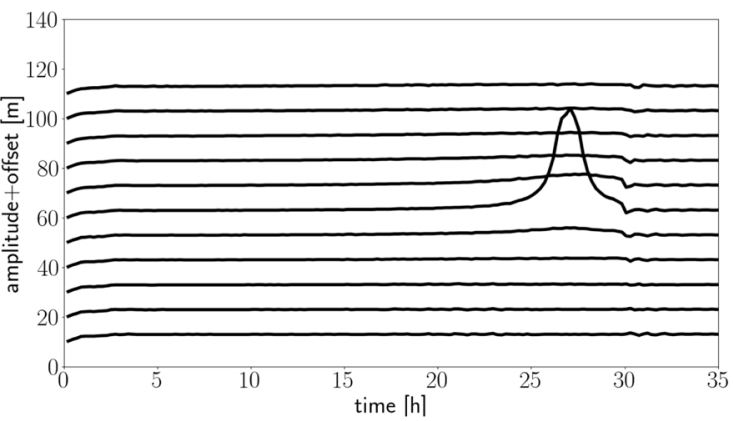

(a) Configuration 1

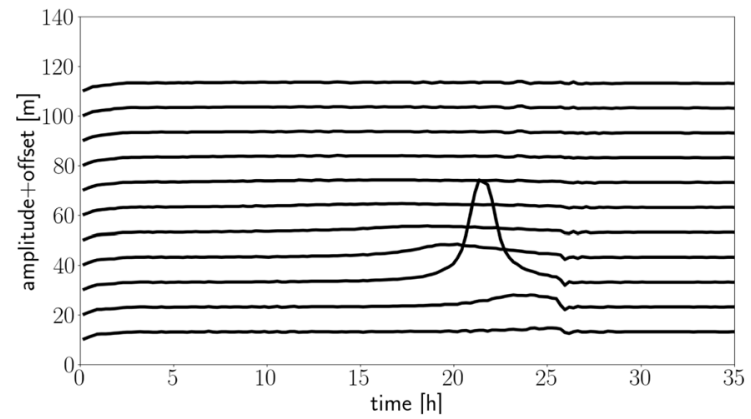

(c) Configuration 2

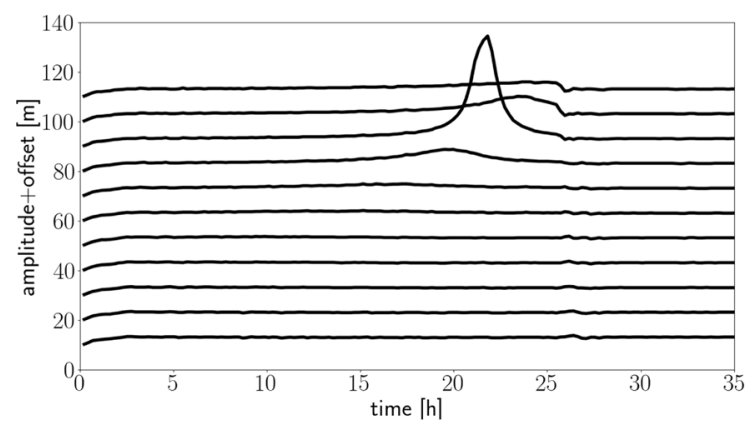

(e) Configuration 3

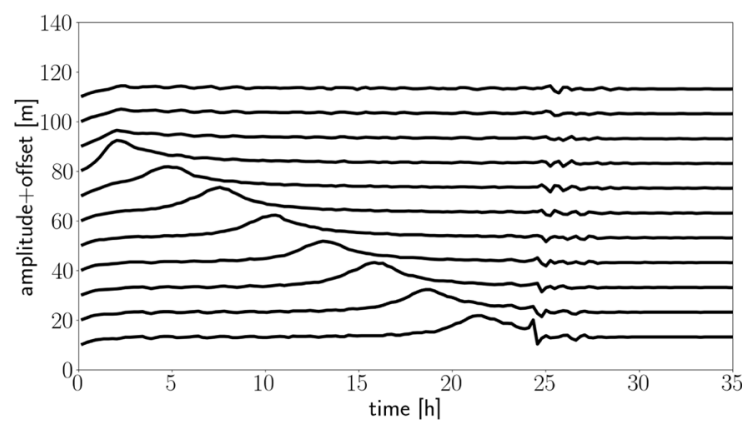

(b) Configuration 4

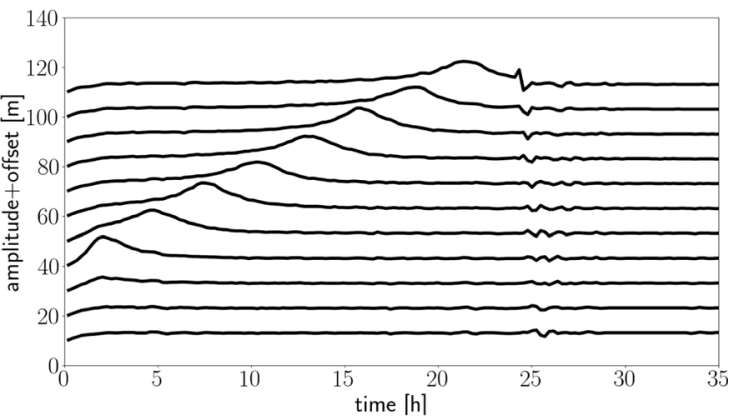

(d) Configuration 5

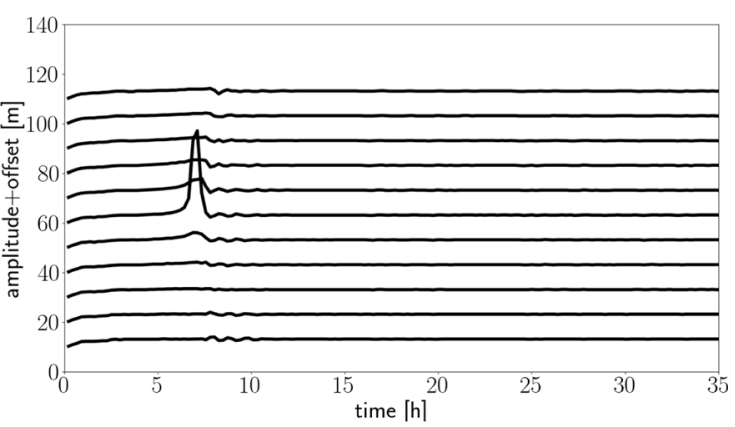

(f) Configuration 6

Fig. 13 Idealised hurricane approaching a linearly sloping coast: waterfall plot of time series at even numbered wave gauges from bottom to top for all six configurations. Amplitudes are in $m$ with an added offset of $10 \cdot k$ for wave gauge $G_{k}$ for all $k$

The wind model (Holland 1980) also gives a corresponding atmospheric pressure

$p_{A}=p_{c}+\left(p_{n}-p_{c}\right) e^{\frac{-A}{B}}$

as is seen from Fig. 2 (right) which shows pressures (dashed line) normalised by $p_{n}-p_{c}$ as well as wind speeds normalised by $\max |v|^{2}$.

\subsubsection{Numerical results}

We ran the simulation with a temporally changing time step, an explicit RK23 time integrator, and a CFL number of 0.2 with a spatial resolution of $\Delta x=10,937.5$ a Manning parameter of $n=0.001$, and the wind drag as in (Weisberg and Zheng 2006). We note that this CFL number differs from the one used
Table 4 Idealised hurricane approaching a linearly sloping coast: maximum wave height $\eta_{\max }$ with respect to ramp up time at gauges $G_{10}, G_{12}$ and $G_{20}$

\begin{tabular}{llllll}
\hline Ramp up time & 15 min & 30 min & 1 hour & 2 hours & 4 hours \\
\hline$\eta_{\max }$ at $G_{10}$ & $41.4 \times 10^{-2}$ & $41.4 \times 10^{-2}$ & $41.7 \times 10^{-2}$ & $41.6 \times 10^{-2}$ & $41.7 \times 10^{-2}$ \\
$\eta_{\max }$ at $G_{12}$ & $6.5 \times 10^{-2}$ & $6.4 \times 10^{-2}$ & $6.5 \times 10^{-2}$ & $6.3 \times 10^{-2}$ & $6.5 \times 10^{-2}$ \\
$\eta_{\max }$ at $G_{20}$ & $2.3 \times 10^{-2}$ & $2.2 \times 10^{-2}$ & $2.2 \times 10^{-2}$ & $2.3 \times 10^{-2}$ & $2.4 \times 10^{-2}$ \\
\hline
\end{tabular}


Table 5 Idealised hurricane approaching a linearly sloping coast: maximum and minimum wave height with respect to different drag coefficients at gauges $G_{10}, G_{12}$ and $G_{20}$

\begin{tabular}{llllll}
\hline Gauge no. & & Weisberg and Zhang & Garrat & Powell & Constant \\
\hline 10 & $\eta_{\max }$ & $41.6 \times 10^{-2}$ & $41.6 \times 10^{-2}$ & $41.6 \times 10^{-2}$ & $41.7 \times 10^{-2}$ \\
& $\eta_{\min }$ & $-10.0 \times 10^{-2}$ & $-9.9 \times 10^{-2}$ & $-9.8 \times 10^{-2}$ & $-9.5 \times 10^{-2}$ \\
12 & $\eta_{\max }$ & $6.3 \times 10^{-2}$ & $6.3 \times 10^{-2}$ & $6.5 \times 10^{-2}$ & $6.5 \times 10^{-2}$ \\
& $\eta_{\min }$ & $-10.5 \times 10^{-2}$ & $-10.5 \times 10^{-2}$ & $-10.4 \times 10^{-2}$ & $-10.0 \times 10^{-2}$ \\
20 & $\eta_{\max }$ & $2.3 \times 10^{-2}$ & $2.3 \times 10^{-2}$ & $2.4 \times 10^{-2}$ & $2.4 \times 10^{-2}$ \\
& $\eta_{\min }$ & $-8.1 \times 10^{-2}$ & $-8.0 \times 10^{-2}$ & $-7.9 \times 10^{-2}$ & $-7.6 \times 10^{-2}$ \\
\hline
\end{tabular}

in all other experiments in this study but was found to be necessary to ensure numerical stability. We then compared the wave signal $\eta(\mathbf{x}, t)=h(\mathbf{x}, t)-3000.0$ at numerical wave gauges $G_{k}$ located at $G_{k} \cdot 10^{-5}=(4.5,-2.5+0.5 k)^{\top}$ for $k=\{0,2,4, \ldots, 20\}$ (see left display of Fig. 12) with the findings in (Mandli 2011) and found overall good agreement. The results are found in Fig. 13. It shows the water wave signal for all six configurations obtained at the numerical wave gauges $G_{k}$ for all even numbered wave gauges with the amplitudes plotted in metre with a vertical offset of $10 \cdot k$ for gauge $G_{k}$ to increase readability. In agreement with the literature (Weisberg and Zheng 2006), we find that the observed flooding is sensitive to the approaching angle. The plots for configurations 2 and 3 in Fig. 13 show significantly different signals at the wave gauges to the left and right of the wave gauge at which the storm made landfall. They differ in shape and arrival time. The general $N$ shape of the largest waves as seen in (Mandli 2011), however, could not be reproduced. Since higher resolution simulations with a halved $\Delta x$ showed the same behaviour, we attribute this effect to the implementation of impermeable boundary conditions in this test case. The rotational direction of the wind velocity, and the impermeable boundaries at $x= \pm 300 \times 10^{3}$ are thought to be responsible for the only approximate symmetries between configurations 2 and 3, as well as 4 and 5. In the absence of Coriolis forcing, the rotational direction impacts the flow,

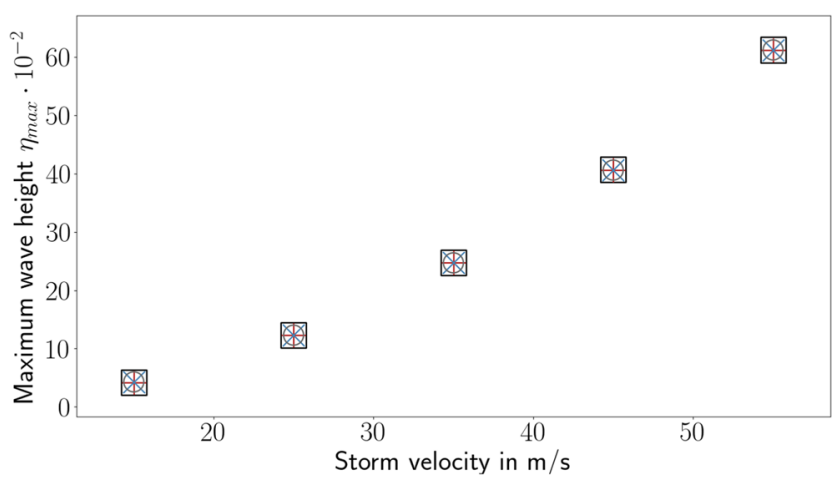

Fig. 14 Idealised hurricane approaching a linearly sloping coast: maximum wave height $\eta_{\max }$ versus maximum wind speed, $\max \mathbf{v}$, for different drag coefficients (black squares - Weisberg and Zheng, red plusses - Powell, blue xs - Garrat and grey circles - constant. Because of the close agreement of obtained $\eta_{\max }$, markers overlay such that the plot of configuration 2 in Fig. 13 is not exactly the same as the plot for configuration 3 mirrored at $y=0$. We furthermore remark that all configurations show small oscillations right after the storm made landfall. This is due to the treatment of the wind stress which was switched off after the storm reached the beach.

\subsubsection{Influence of ramp up times and robustness}

Numerical models often require a gentle ramping up of source terms in order to reduce spurious oscillations and to allow for a robust computation. In our simulations, we ramped up the wind stress $\tau$ and pressure $p$ using an exponential blending in time. For early times, $t \leq t_{r u}$ this filter $\mathcal{F}$ takes on the form

$\mathcal{F}(\phi)=\phi \mathrm{e}^{-\left(\frac{t-t_{r u}}{c_{f} t_{r u}}\right)^{2}}$,

with $\phi$ the quantity that is to be started and $c_{f}$ a tunable coefficient.

The storm only starts travelling towards the coast with angle $\theta$ after the ramp up time $t_{r u}$ is reached. Before, it is kept at its starting position, so that the wind and pressure fields are slowly ramped up until they reach their full strength. We ran configuration 1 with five different ramp up times between $15 \mathrm{~min}$ and 4 hours (see also Table 4) to test the robustness of the results. Ideally, we would like $t_{r u}$ to be as small as possible to save computing time but large enough to not pollute numerical results. We observe that for all times between $15 \mathrm{~min}$ and 4 hours we get robust

Table 6 Idealised hurricane approaching a linearly sloping coast: Holland parameters for storms of different sizes/varying RMW

\begin{tabular}{llllll}
\hline Configuration & $\Delta p$ & $\begin{array}{l}\max |\boldsymbol{v}| \\
\text { in } \mathrm{m} \mathrm{s}^{-1}\end{array}$ & $\begin{array}{l}\text { RMW in } \\
\mathrm{km}\end{array}$ & $A$ & $B$ \\
\hline $1 \mathrm{a}$ & 4600.0 & 50.0 & 10.0 & 50.11 & 1.7 \\
$1 \mathrm{~b}$ & 4600.0 & 50.0 & 20.0 & 162.313 & 1.7 \\
$1 \mathrm{c}$ & 4600.0 & 50.0 & 47.0 & 695.931 & 1.7 \\
$1 \mathrm{~d}$ & 4600.0 & 50.0 & 75.0 & 1533.171 & 1.7 \\
\hline
\end{tabular}


Table 7 Idealised hurricane approaching a linearly sloping coast: Maximum wave height for different storm sizes at wave gauge $G_{10}$

\begin{tabular}{clll}
\hline $\begin{array}{l}\text { Configuration } \\
1 \mathrm{a}\end{array}$ & $\begin{array}{l}\text { Configuration } \\
1 \mathrm{~b}\end{array}$ & $\begin{array}{l}\text { Configuration } \\
1 \mathrm{c}\end{array}$ & $\begin{array}{l}\text { Configuration } \\
1 \mathrm{~d}\end{array}$ \\
\hline$\eta_{\max } 38.6 \times 10^{-2}$ & $48.4 \times 10^{-2}$ & $55.2 \times 10^{-2}$ & $63.4 \times 10^{-2}$ \\
\hline
\end{tabular}

numerical results. Furthermore, as is seen from Table 4, the maximum wave height does not show a lot of variation depending on different ramp up times and the maximum variation is found to be $3 \times 10^{-3}$. Wave gauge signals, however, detect a small wave at times $t \approx 1 \mathrm{~h}$ for ramp up times below 2 hours. Hence, we chose a ramp up time of $t_{r u}$ of 2 hours for this study.
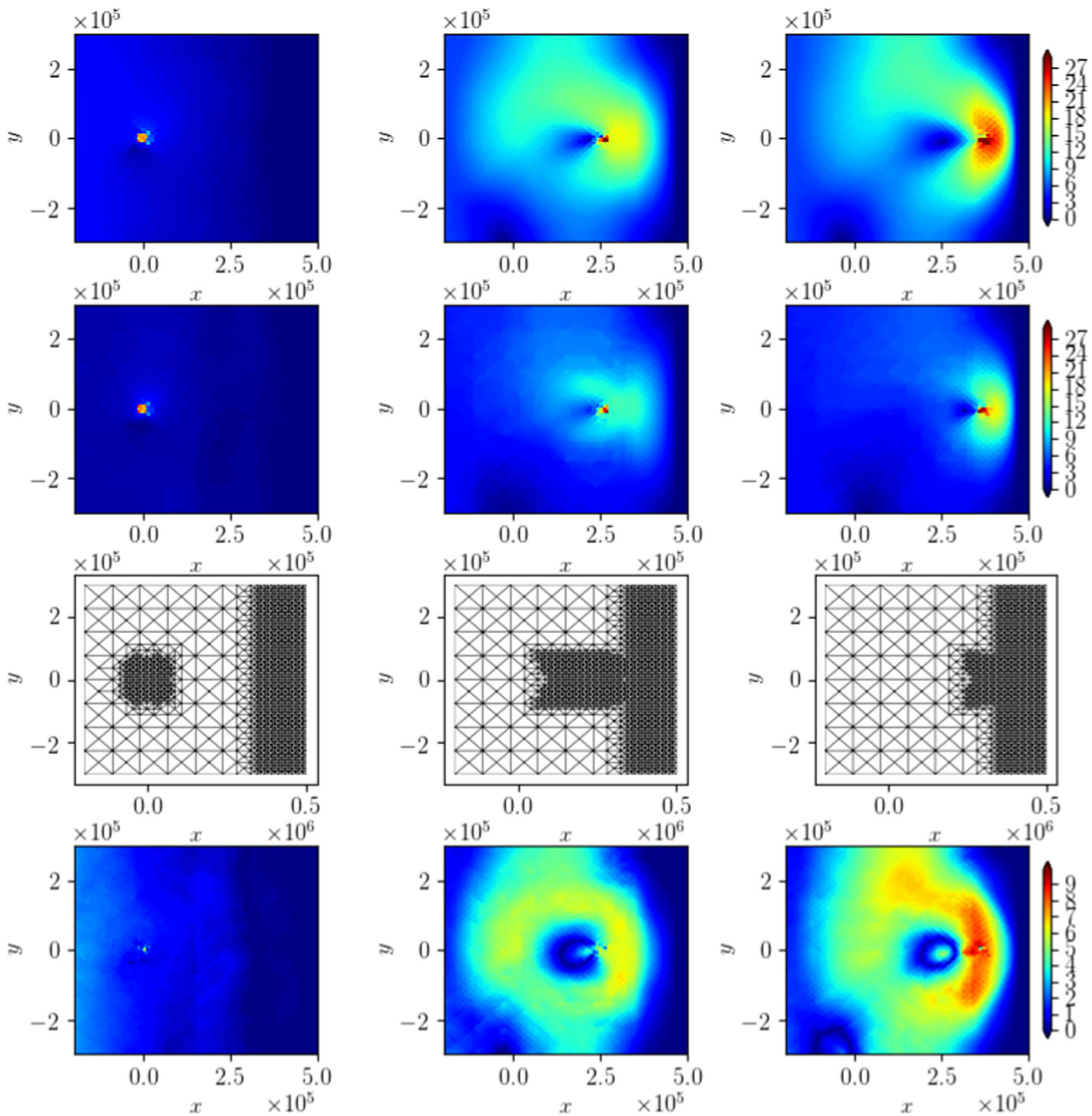

Fig. 15 Idealised hurricane approaching a linearly sloping beach: Simulation results for configuration 1 at time $t=0$, midway to landfall and close to landfall (left to right). Depicted are the current magnitudes of

the uniform (top) and adaptive (top middle) simulation; the adaptive meshes (bottom middle) and absolute difference between both simulations (bottom) 

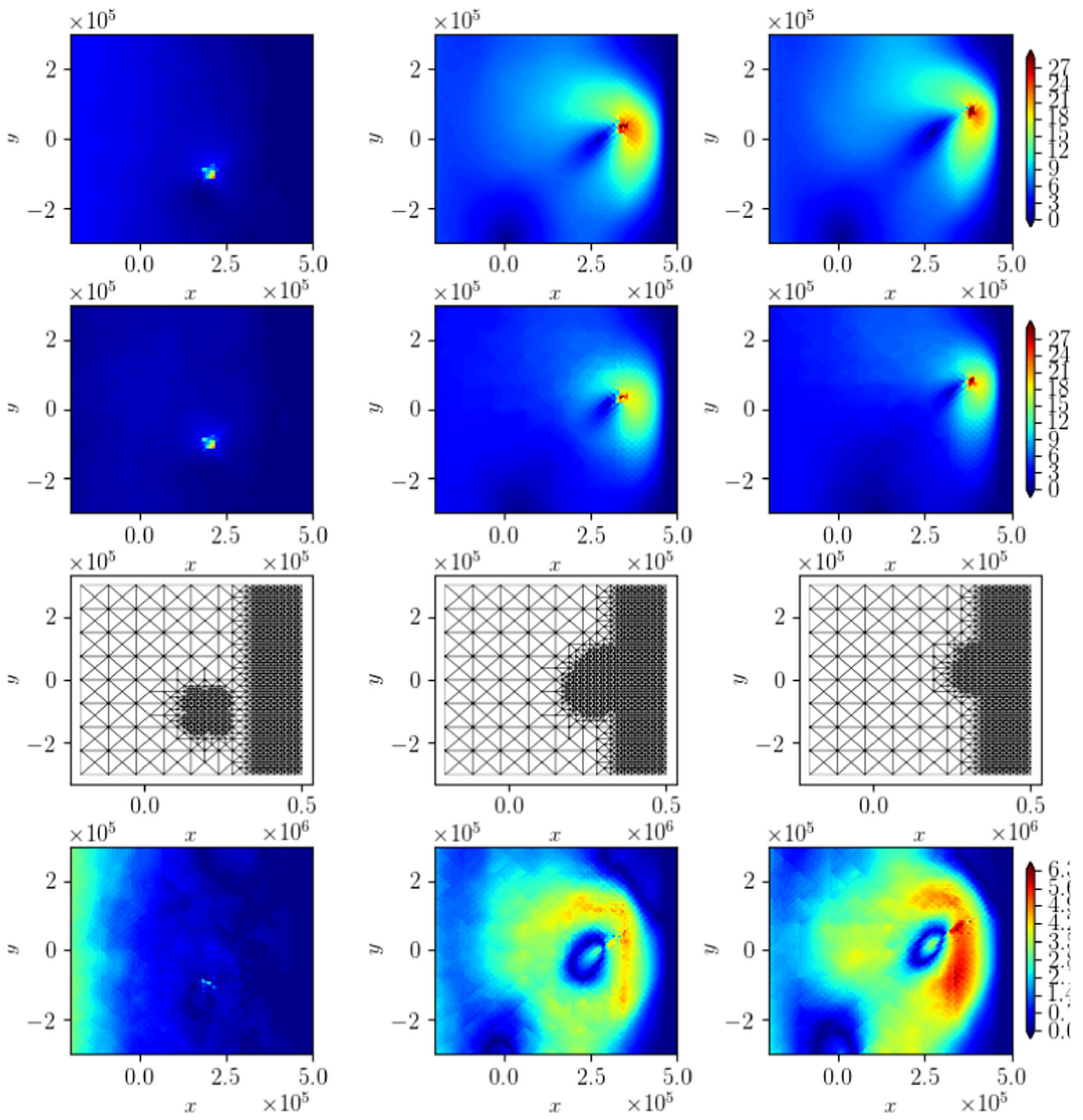

Fig. 16 Idealised hurricane approaching a linearly sloping beach: Simulation results for configuration 2 at time $t=0$, midway to landfall and close to landfall (left to right). Depicted are the current magnitudes of

the uniform (top) and adaptive (top middle) simulation; the adaptive meshes (bottom middle) and absolute difference between both simulations (bottom)

\subsubsection{Sensitivity with respect to the wind drag parameter $c_{d}$}

Modelling the energy transfer from the atmosphere to the ocean surface is accomplished through a wind drag parameter $c_{d}$ (a source term; see also Eq. (8) that couples the external wind field to the hydrodynamic model). As described in Sect. 2.1.4, several wind drag parameterisations have been developed in the literature. Exemplarily, we tested the set of four parameterisations described in Sect. 2.1.4 for configuration 1 in order to determine their influence on maximum wave heights at wave gauges $G_{k}$ which we assume a good indicator for wave run-up at the coast. Table 5 shows maximum and minimum wave heights for the original configuration 1 at selected wave gauges closest to the storm's landfall. We repeated the simulation with all four different wind drag models and observe merely minor differences of the order of at most $0.5 \times$ 

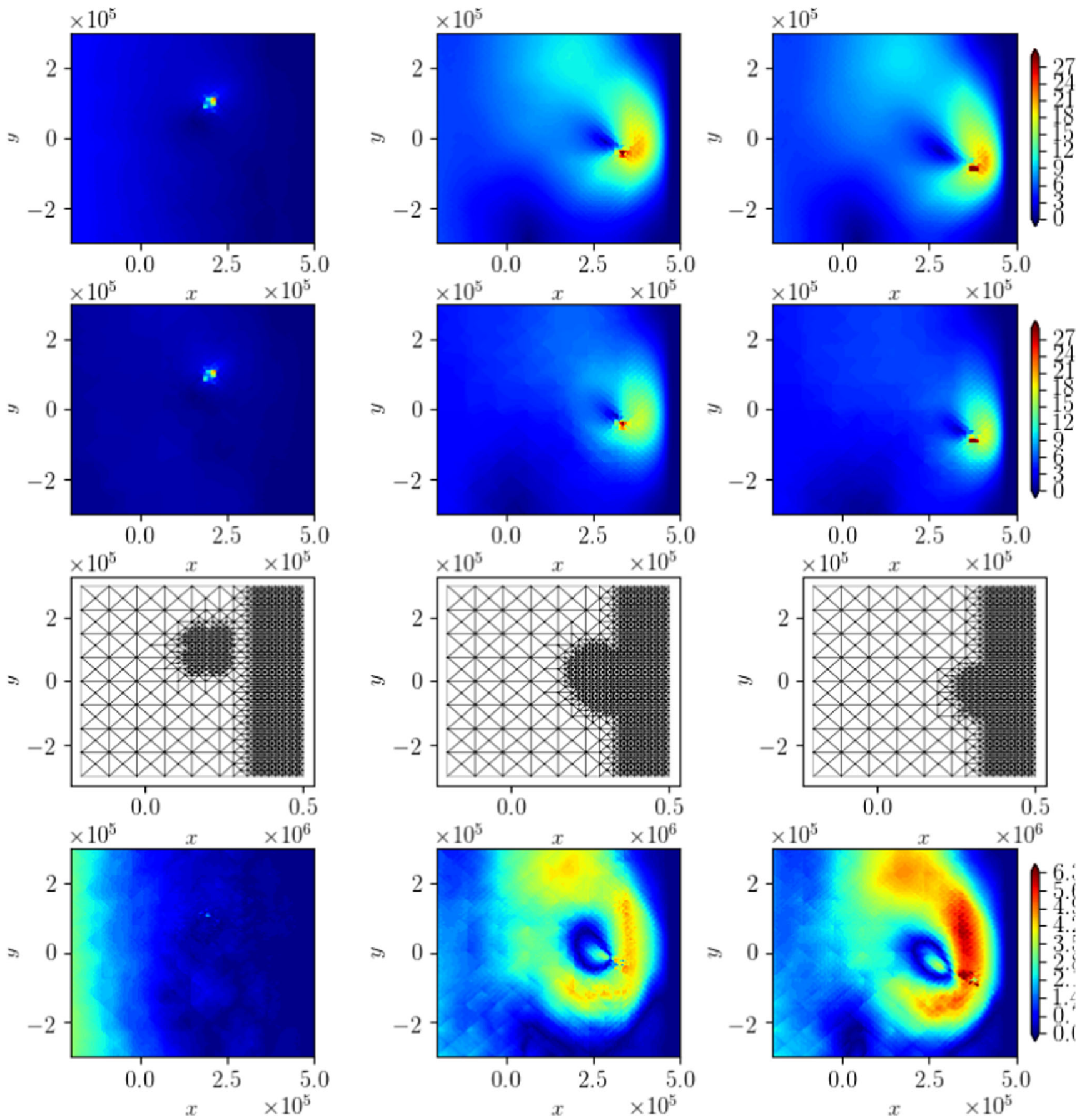

Fig. 17 Idealised hurricane approaching a linearly sloping beach: Simulation results for configuration 3 at time $t=0$, midway to landfall and close to landfall (left to right). Depicted are the current magnitudes of

the uniform (top) and adaptive (top middle) simulation; the adaptive meshes (bottom middle) and absolute difference between both simulations (bottom)

$10^{-2}$. The parameter $c_{d}=c_{d}(\mathbf{v})$ depends on the wind speed. Therefore, we ran configuration 1 with varying maximum wind speeds max $|\boldsymbol{v}| \in\{15,25,35,45,55\}$ for every wind drag model. As shown in Fig. 14, we see that the differences in maximum wave heights $\eta_{\max }$ are negligible. In fact, they are, again, of the order of at most $0.5 \times 10^{-2}$. Hence, we conclude that in an idealised model such as the one presented in this manuscript different wind drag models do not lead to significantly different results. This can be explained by the form of the wind stress $\tau=\rho_{a} c_{d}\|\mathbf{v}\|_{2} \mathbf{v}$. Using the selected parameterisations, $c_{d}$ will differ at most by a factor of 2 in very localised regions of the storm, which does not lead to a significant increase or decrease in the observed wave heights close to the coast. 

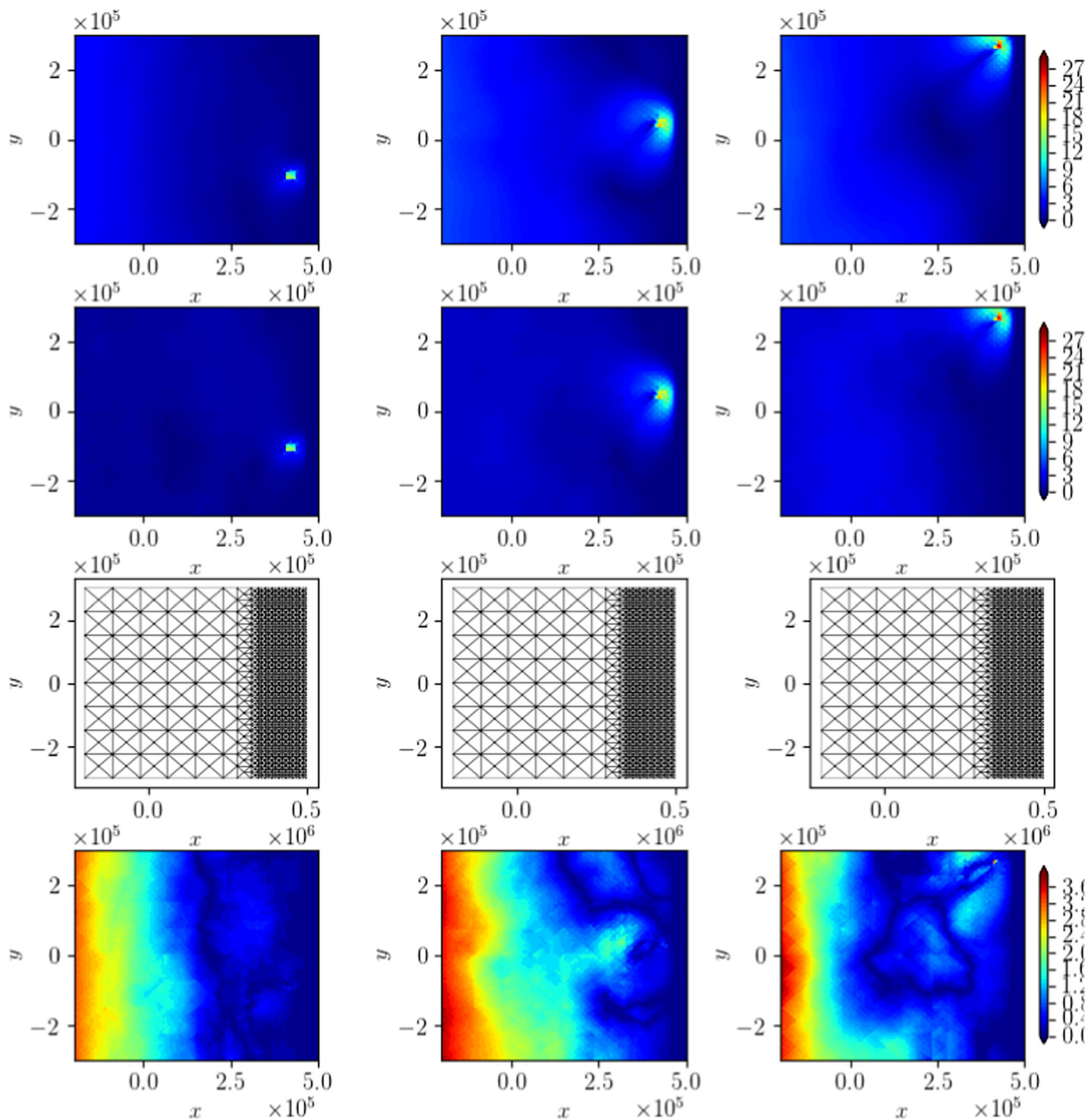

Fig. 18 Idealised hurricane approaching a linearly sloping beach: Simulation results for configuration 4 at time $t=0$, midway to landfall and close to landfall (left to right). Depicted are the current magnitudes of

the uniform (top) and adaptive (top middle) simulation; the adaptive meshes (bottom middle) and absolute difference between both simulations (bottom)

\subsubsection{Influence of storm size}

The size of a hurricane plays an important role in the observed flooding. For reasonable storm sizes, a variability of about $30 \%$ in observed surge is reported (Irish et al. 2008). In the wind model (Holland 1980), the shape and size of the storm depend on the shape parameters $A$ and $B$. These, in turn, depend on the radius of maximum winds (RMW), the difference between ambient and central pressure $\Delta p=p_{n}-p_{c}$, the air density $\rho_{a}$ and the maximum wind speeds as shown in Eq. (11). Assuming storm conditions that are representative for the 2017 hurricane Ophelia, we simulate configuration 1 as described above and vary the radius of maximum winds. According to (Hsu and Yana 1998), the average radius of maximum winds of hurricanes is 

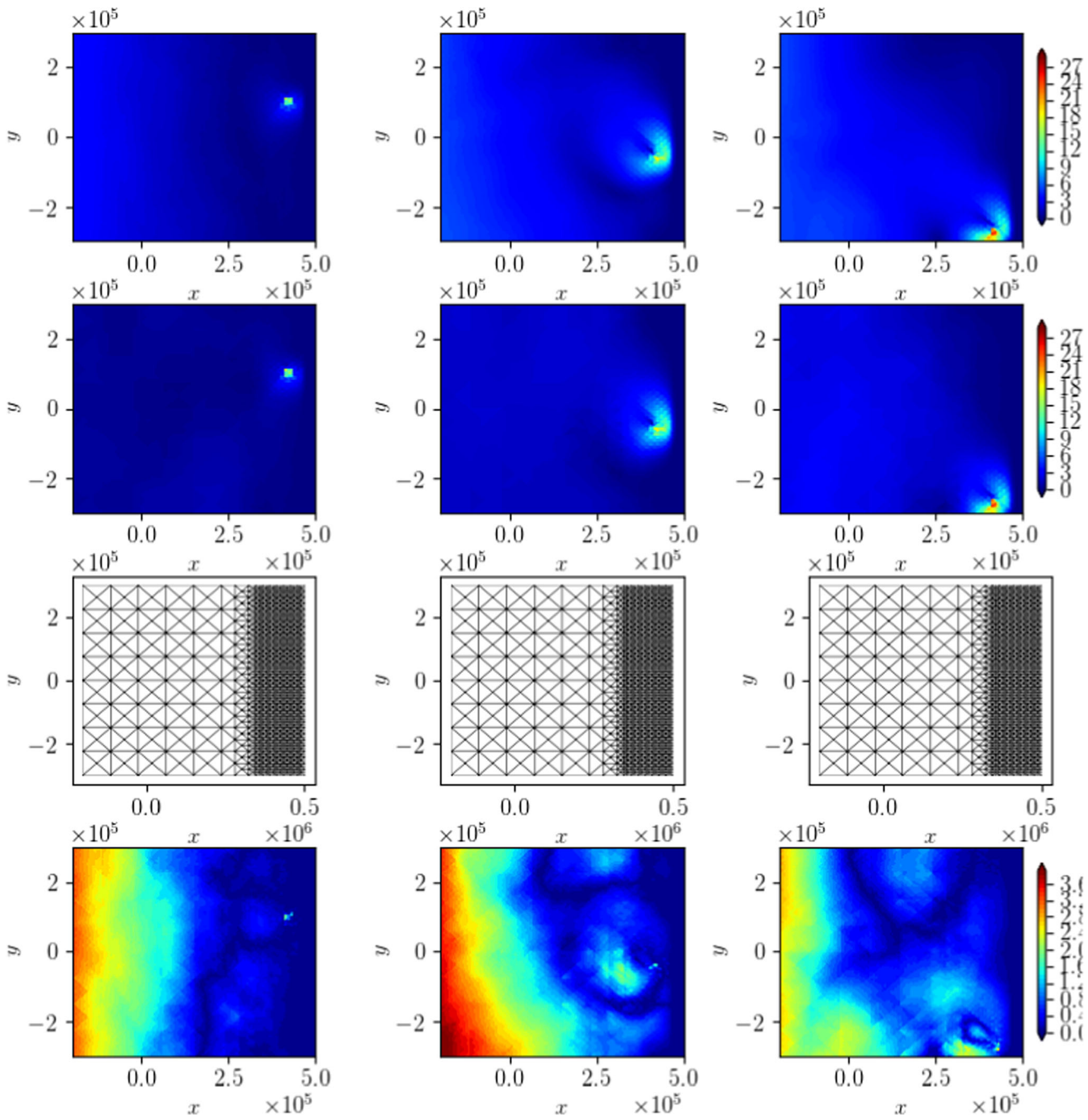

Fig. 19 Idealised hurricane approaching a linearly sloping beach: Simulation results for configuration 5 at time $t=0$, midway to landfall and close to landfall (left to right). Depicted are the current magnitudes of

the uniform (top) and adaptive (top middle) simulation; the adaptive meshes (bottom middle) and absolute difference between both simulations (bottom)

$47 \mathrm{~km}$, with a standard deviation of $27 \mathrm{~km}$ which is why we chose to run simulations with the radii stated in the Table 6. As can be seen, only parameter $A$ varies with varying RMW if all other conditions are kept fixed as it describes the radial scaling on the RMW and the location of the maximum wind relative to the origin. We measured the maximum wave height at wave gauge $G_{10}$ - the location at which the synthetic storm made landfall - and will hence record the maximum surface elevation $\eta_{\max }$. The results are depicted in Table 7 . We see that with this simple parameterisation, we achieve measured maximum wave heights with a variability of about $39 \%$. Given that we tested with parameters resembling hurricane Ophelia for the most part, we conclude that we are within the range of variability that was found in (Irish et al. 2008). 

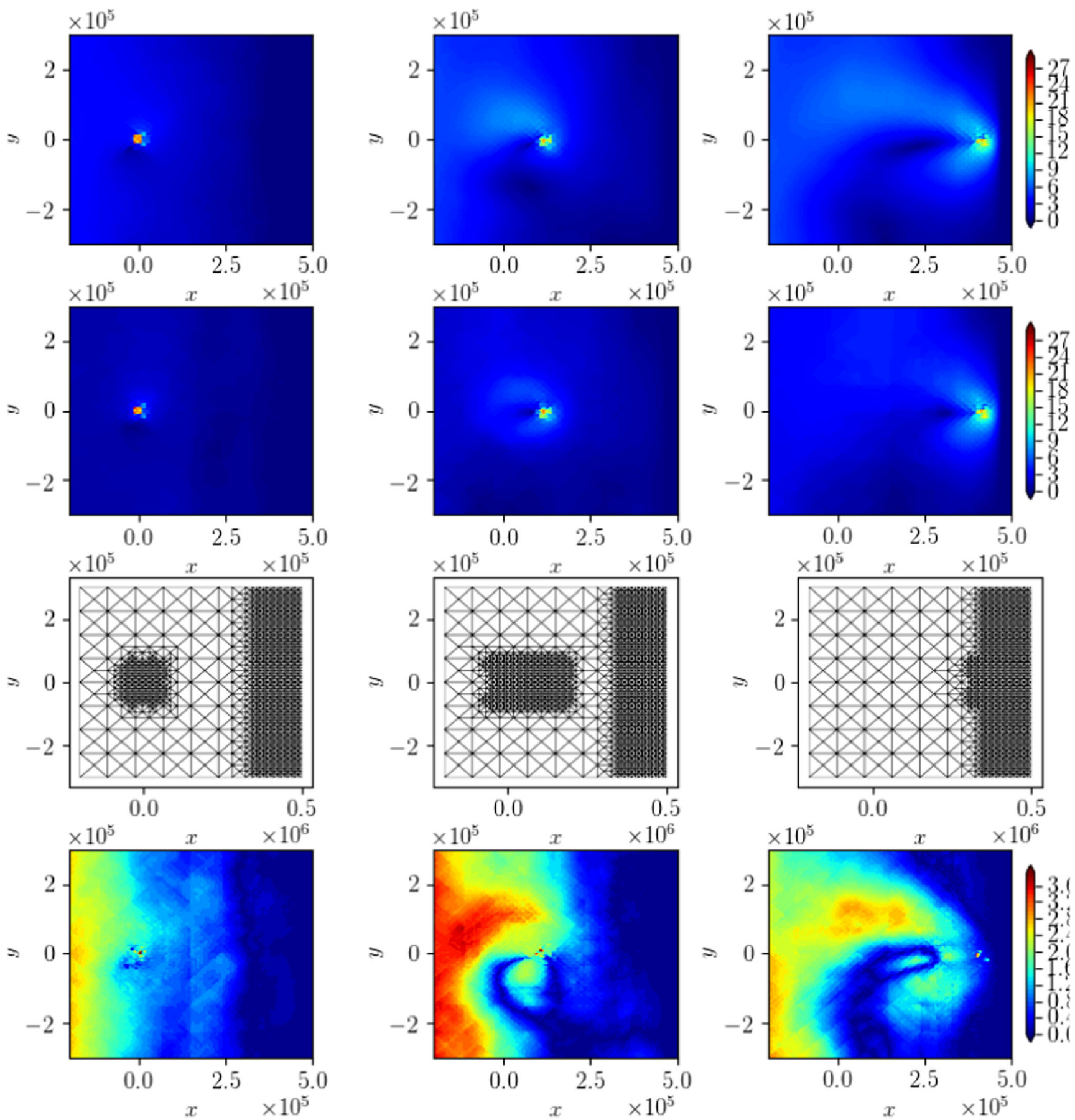

Fig. 20 Idealised hurricane approaching a linearly sloping beach: Simulation results for configuration 6 at time $t=0$, midway to landfall and close to landfall (left to right). Depicted are the current magnitudes of

the uniform (top) and adaptive (top middle) simulation; the adaptive meshes (bottom middle) and absolute difference between both simulations (bottom)

\subsubsection{Adaptive simulations}

Dynamically changing non-uniform meshes as described in Sect. 2.2 are ideal for simulating localised phenomena at a reduced computational cost. Since storm wave heights are strongly influenced by changes in bathymetry as well as the

size and strength of a storm, we define a refinement indicator $\eta_{\Omega_{i}}$ to take both of them into account for every element $\Omega_{i}$ :

$\eta_{\Omega_{i}}=|\nabla b|_{\Omega_{i}, 1}+|\boldsymbol{v}|_{\Omega_{i}, 2}$.

Using the indicator (Eq. (12)), we achieve a refinement of the beach or bathymetry gradient as well as the storm position 


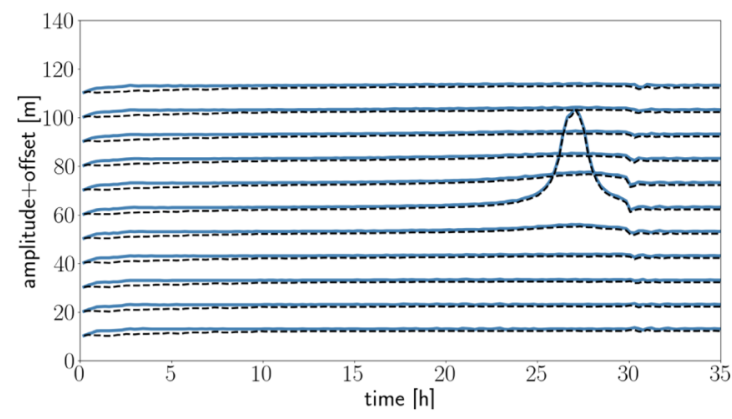

(a) Configuration 1

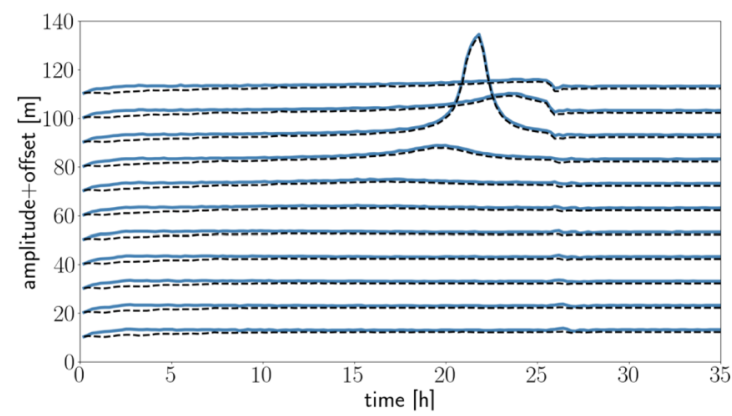

(c) Configuration 3

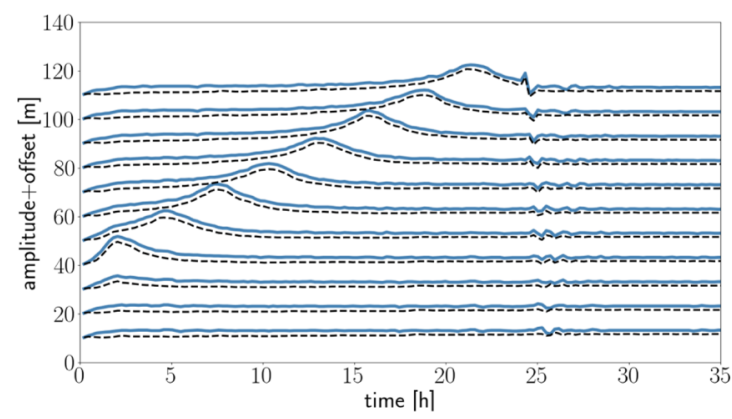

(e) Configuration 5

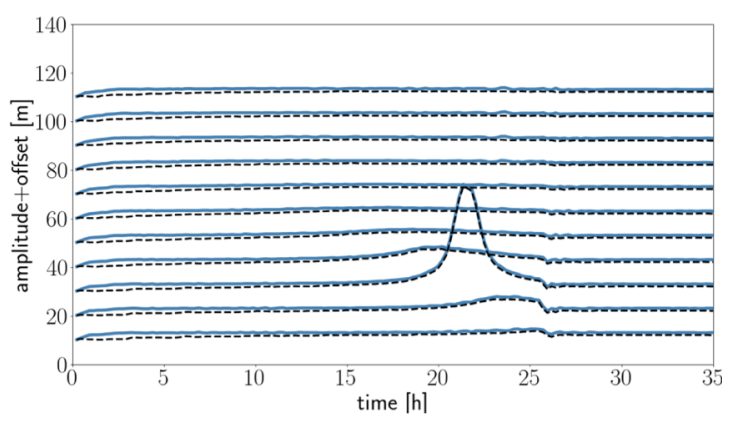

(b) Configuration 2

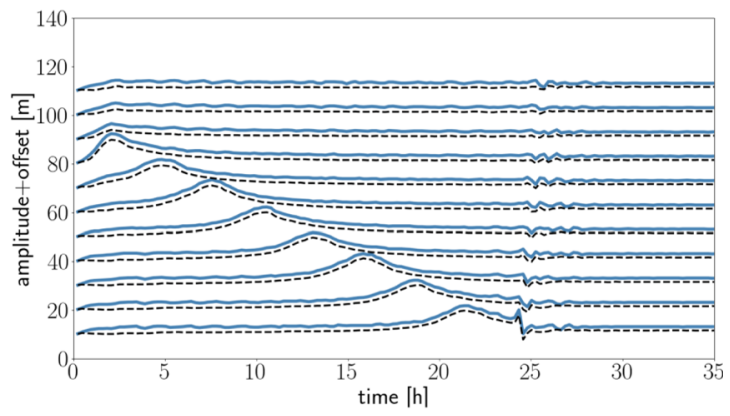

(d) Configuration 4

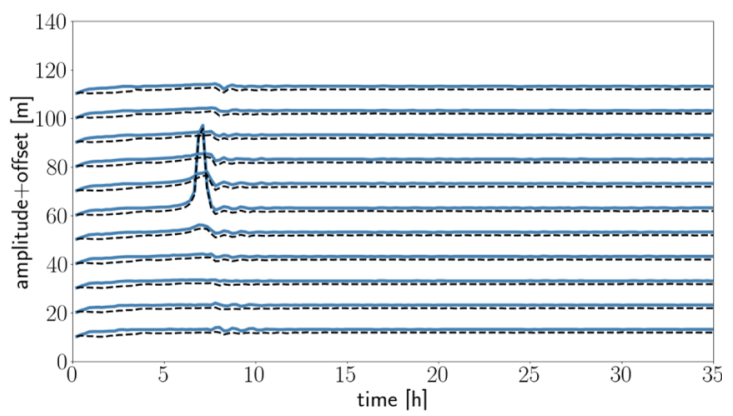

(f) Configuration 6

Fig. 21 Idealised hurricane approaching a linearly sloping beach: Waterfall plot of wave gauges $G_{k}$ over time for uniform (black dashed line) and adaptive (blue solid line). Amplitudes are in $\mathrm{m}$ with an offset of $10 \cdot k \mathrm{~m}$ for wave gauge $G_{k}$

(see Figs. 15, 16, 17, 18, 19 and 20). Figures 15, 16, 17, 18, 19 and 20 show numerical results for configurations $1-6$ on an adaptive and a uniform mesh. Plotted are the non-uniform meshes and currents at the initial time, about halfway to landfall and close to landfall. We can see that the adaptive and uniform simulations yield comparable results with small errors in areas of high resolution and slightly larger errors outside. The refined region in the adaptive simulation comprises the entire area close to the coast as well as the cyclone and with time we see that the refined area resolving the cyclone moves with the storm. Some numerical error is observed which we attribute to the choice of the heuristic refinement indicator which captures physical features that only indirectly correlate with numerical error and model sensitivities. This,
Fig. 22 Idealised dam break: Domain description with inlet depicted by a diamond

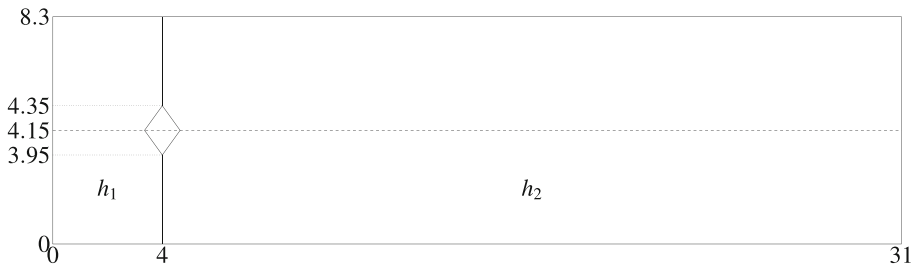


Fig. 23 Idealised dam break: Macro-triangulation

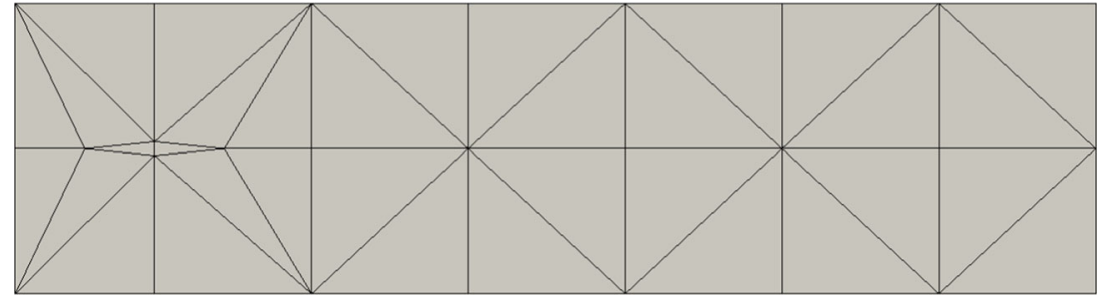

however, does not impact the measured wave heights near the coast. The maximum wave heights measured at gauges $G_{k}$ are depicted in Fig. 21 and show good agreement between the adaptive and uniform simulations. Hence, since our interest lies in the wave gauge signal, the refinement indicator seems suitable. Although dynamic mesh refinement adds computational overhead, this was not found to be significant. For configuration 1 (Fig. 15), the uniform simulation comprised 8192 elements, while the adaptive simulation only used on average: 2714 (and a maximum 3074) elements - a reduction to at least $37.52 \%$ of the elements (on average $33.13 \%$ ). In terms of run time, the adaptive simulation used about $31.33 \%$ of the computational time by yielding quantitatively the same result in terms of measured wave heights. A similar behaviour can be observed for all configurations.

\subsection{Idealised dam break}

In a rectangular domain $\Omega=[0,31] \times[0,8.3]$ that is separated by a wall at $x=4$ (see also Fig. 22), two different constant water levels are prescribed as

$h(\mathbf{x}, 0)=\left\{\begin{array}{ll}h_{1} & x \leq 4 \\ h_{2} & \text { otherwise }\end{array}\right.$,

with $h_{1}=0.6$ and $h_{2}=0.05$. We furthermore assume zero initial velocities $\mathbf{u}(\mathbf{x}, 0)=\mathbf{0}$. At time $t=0$, a dam break is simulated by removing the wall between $3.95 \leq y \leq 4.35$ as indicated by the diamond in Fig. 22.

To preserve the space-filling curve (SFC) ordering of the elements and to resolve the narrow inlet, we used the macrotriangulation depicted in Fig. 23 as an initial mesh for the uniform as well as adaptive mesh refinement. This comes at the expense of the time step being limited by the narrowest element which we will further comment on later in this paragraph.

We ran the uniform simulation with a CFL number of 0.3 which resulted in a time step of about $\Delta t=5 \cdot 10^{-4}$ and a total number of elements of 69,632 which corresponds to a 12 times uniform refinement of the mesh depicted in Fig. 23. Snapshots of the numerical solution at times $t=0,2$, and 4 on a uniform and adaptively refined mesh are found in Fig. 24. We observe that a large wave develops from the inlet and starts travelling across the shallow part of the domain. Using the fine resolution uniform simulation as a reference solution, we ran an adaptive simulation, refining according to momentum maxima, i.e. using a refinement indicator $\eta_{\Omega_{i}}(t)=\max _{\mathbf{x} \in \Omega_{i}}|\mathbf{u}(\mathbf{x}, t)|$. The adaptive mesh comprised a finest resolution identical to the uniform simulation but a coarsest resolution resulting from only 8 times refining the macro-triangulation. The results on the adaptive and uniform mesh are virtually indistinguishable. We can see that the fine mesh area follows the emerging wave
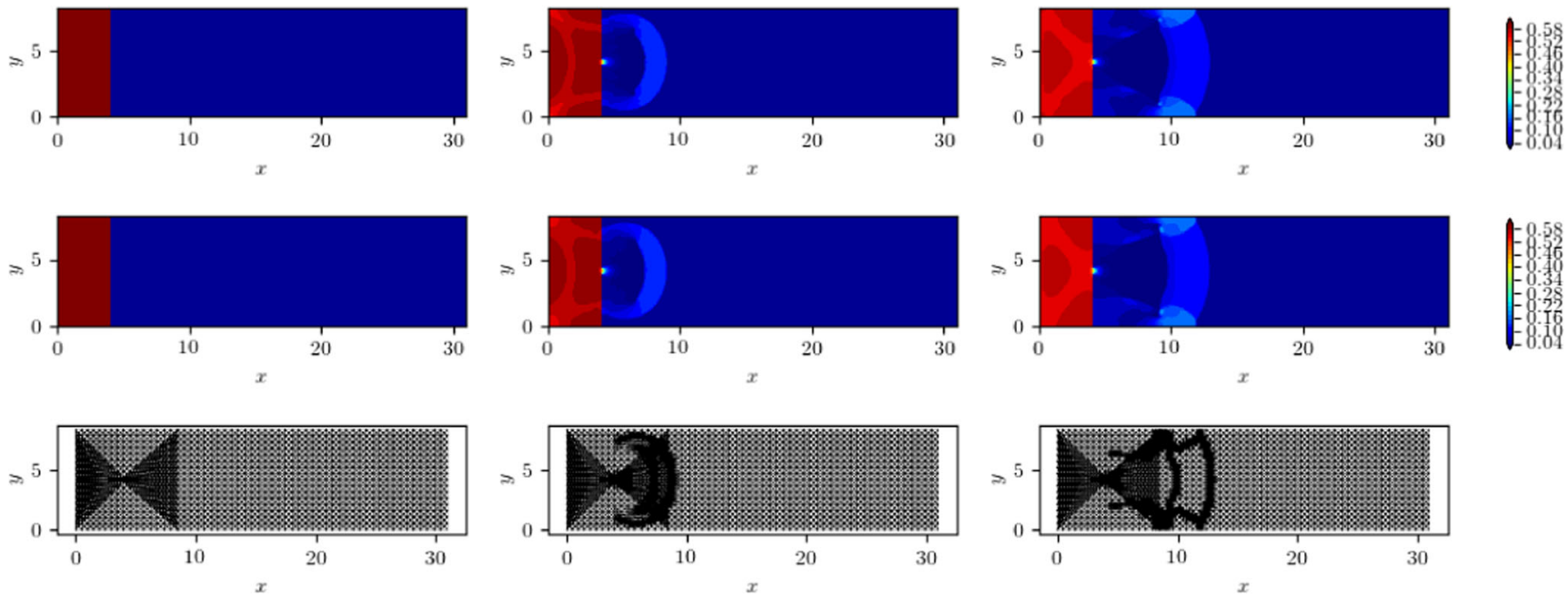

Fig. 24 Idealised dam break: Snapshots of numerical solution on uniform (top) and adaptive mesh (middle) at times $t=0,2$, and $4 \mathrm{~s}$ (left to right), and corresponding adaptive mesh (bottom) 
and only regions are refined that experience fluid movement. Due to the not uniform macro-triangulation, the region around the inlet is highly refined, ensuring that flows are accurately captured. The adaptive mesh comprised on average 8437 elements which is about $12 \%$ of the number of elements of the uniform simulation. In terms of computing time, the adaptive simulation only took $30.5 \%$ of the computing time for the uniform simulation-leading to a cost reduction of almost $70 \%$.

\section{Conclusions and future work}

In this study, we have developed a discontinuous Galerkin model on a dynamically adaptive triangular mesh that solves the fully 2D non-linear shallow water equations for the simulation of coastal flooding and idealised storm surges.

Numerical test cases that we believe to be a good basis for a test suite that might be useful for storm surge modellers demonstrate that the obtained model is inundation stable due to advanced slope limiting techniques (Vater et al. 2019) and maintains important conservation properties such as mass as well as integrated fluxes as described in Sect. 3.1 for the simulation of a hypothetical embayment. Moreover, a steady state is achieved numerically in Sect. 3.2 in which a balance between pressure gradients and wind stress is simulated. In Sect. 3.3, we show the robustness of the wind forcing and the effect of Coriolis forcing on wind-induced circulation. We furthermore see that the wind forcing is robust with respect to ramp up times; hence, no spurious artefacts are introduced.

Finally, in Sect. 4.1, we show the capability of the model to simulate idealised hurricane storm surge using the wind parameterisation (Holland 1980), hence making it suitable for simulation of realistic hurricanes such as the 2008 Atlantic hurricane Ike or the 2017 hurricane Ophelia. A sensitivity analysis furthermore reveals that the model is not sensitive to the choice of wind drag parameterisation or storm ramp up time. The observed variability of maximum wave heights (and therewith wave run up) with varying RMW confirms previously published studies, underlining the capability of the model to yield realistic results. Most notably, using dynamically adaptive meshes, we obtain virtually the same signal at wave gauges close to the beach at significantly less computational cost: The reduction of computing time was measured to be up to $70 \%$. Overall, this is to demonstrate that the developed model is suitable for the simulation of idealised hurricane storm surge and shows a satisfactory robustness and accuracy as well as adaptive mesh capabilities that help reduce computing costs significantly.

The same reduction of about $70 \%$ could also be shown for the idealised dam break problem in which we showed that the mesh is accurately following the emerging waves.
In this study, we have dealt with a number of idealised test cases and demonstrated the model's potential to use an adaptive mesh for the simulation of hurricane storm surge. The application of the presented model to more realistic data is beyond the scope of this paper and will be left for future research. The results presented, however, allow the conclusion that the combination of dynamically adaptive mesh refinement with a DG discretisation significantly increase the potential practicality of the model and can be seen as a first indication of DG methods being a useful tool for the application to hurricane storm surge modelling.

Acknowledgements We wish to acknowledge the DJEI/DES/SFI/HEA Irish Centre for High-End Computing (ICHEC) for the provision of computational facilities and support.

Software availability The presented numerical model has been implemented in Fortran90 and extensively tested using the gfortran compiler. The latest published version can be made available without warranty to registered users at amatos.info.

Funding information We received funding from Science Foundation Ireland (SFI) under the research project "Understanding Extreme Nearshore Wave Events through Studies of Coastal Boulder Transport" (14/US/E3111) as well as by the Irish Research Council (IRC) under the research project "NIMBUS ${ }^{3}$ : Next-Generation Integrated Model for Better and Unified Storm Surge Simulations" (GOIPD/2018/248).

\section{Compliance with ethical standards}

Conflict of interest The authors declare that they have no conflict of interest.

Open Access This article is licensed under a Creative Commons Attribution 4.0 International License, which permits use, sharing, adaptation, distribution and reproduction in any medium or format, as long as you give appropriate credit to the original author(s) and the source, provide a link to the Creative Commons licence, and indicate if changes were made. The images or other third party material in this article are included in the article's Creative Commons licence, unless indicated otherwise in a credit line to the material. If material is not included in the article's Creative Commons licence and your intended use is not permitted by statutory regulation or exceeds the permitted use, you will need to obtain permission directly from the copyright holder. To view a copy of this licence, visit http://creativecommons.org/licenses/by/4.0/.

\section{References}

Abdi DS, Giraldo FX, Constantinescu EM, Carr LE, Wilcox LC, Warburton TC (2019) Acceleration of the IMplicit-EXplicit nonhydrostatic unified model of the atmosphere on manycore processors. Int J High Perform Comput Appl 33(2):242-267. https:// doi.org/10.1177/1094342017732395

Antonopoulos DC, Dougalis VA (2016) Galerkin-finite element methods for the shallow water equations with characteristic boundary conditions. IMA J Numer Anal 37(1):266-295. https://doi.org/10.1093/ imanum/drw017

Barnard PL, Erikson LH, Foxgrover AC, Hart JAF, Limber P, O’Neill AC, van Ormondt M, Vitousek S, Wood N, Hayden MK, Jones JM (2019) Dynamic flood modeling essential to assess the coastal 
impacts of climate change. Sci Rep 9(4309). https://doi.org/10.1038/ s41598-019-40742-z

Beckers S, Behrens J, Wollner W (2019) Duality based error estimation in the presence of discontinuities. Appl Numer Math 144:83-99. https://doi.org/10.1016/j.apnum.2019.05.016

Behrens J (2005) Multilevel optimization by space-filling curves in adaptive atmospheric modeling. In: Hülsemann F, Kowarschik M, Rüde U (eds) Frontiers in simulation - 18th Symposium on Simulation Techniques. SCS Publishing House, Erlangen, pp 186-196

Behrens J (2006) Adaptive atmospheric modeling. Springer

Behrens J, Bader M (2009) Efficiency considerations in triangular adaptive mesh refinement. Philos Trans R Soc A Math Phys Eng Sci 367(1907):4577-4589. https://doi.org/10.1098/rsta.2009.0175

Behrens J, Rakowsky N, Hiller W, Handorf D, Läuter M, Päpke J, Dethloff K (2005) amatos: Parallel adaptive mesh generator for atmospheric and oceanic simulation. Ocean Model 10(1-2):171-183. https://doi.org/10.1016/j.ocemod.2004.06.003

Beisiegel N (2014) High-order adaptive discontinuous Galerkin inundation modeling. Ph.D. thesis, Universität Hamburg. URL http://ediss. sub.uni-hamburg.de/volltexte/2014/7036

Beisiegel N, Dias F (2019) Representation of Atlantic hurricanes in MÉRA data and their effect on coastal waves. In: Gleeson E, Whelan E (eds) MÉRA Workshop Proceedings. Met Éireann URL http://edepositireland.ie/handle/2262/91341

Casulli V, Stelling G (1998) Numerical simulation of 3D quasi-hydrostatic, free-surface flows. J Hydraul Eng 124:678-686

Courant R, Friedrichs K, Lewy H (1928) Über die partiellen Differenzengleichungen der mathematischen Physik. Math Ann 100(1):32-74. https://doi.org/10.1007/BF01448839

Danilov S (2013) Ocean modelling on unstructured meshes. Ocean Model 69:195-210

Davis JR, Sheng YP (2003) Development of a parallel storm surge model. Int J Numer Meth Fluids 42:549-580

Dawson C, Kubatko EJ, Westerink JJ, Trahan C, Mirabito C, Michoski C, Panda N (2011) Discontinuous Galerkin methods for modeling hurricane storm surge. Adv Water Resour 34(9):1165-1176. https://doi. org/10.1016/j.advwatres.2010.11.004

Farrell P, Ham D, Funke S, Rognes M (2013) Automated derivation of the Adjoint of high-level transient finite element programs. SIAM J Sci Comput 35(4):C369-C393. https://doi.org/10.1137/120873558

Garrat JR (1977) Review of drag coefficients over oceans and continents. Mon Weather Rev 105:915-929

Gottlieb S, Ketcheson D, Shu CW (2011) Strong stability preserving Runge-Kutta and multistep time Discretizations. World Sci. https:// doi.org/10.1142/7498 URL https://www.worldscientific.com/doi/ abs $/ 10.1142 / 7498$

Hermann V, Käser M, Castro CE (2011) Non-conforming hybrid meshes for efficient 2D wave propagation using the discontinuous Galerkin method. Geophys J Int 184(2):746-758

Hesthaven JS, Warburton T (2008) Nodal discontinuous Galerkin methods: algorithms, analysis, and applications. Springer

Holland GJ (1980) An analytic model of the wind and pressure profiles in hurricanes. Mon Weather Rev 108:1212-1218

Hsu SA, Yana Z (1998) A note on the radius of maximum winds for hurricanes. J Coast Res 12(2):667-668 Coastal Education \& Research Foundation, Inc

Ip JTC, Lynch DR, Friedrichs CT (1998) Simulation of estuarine flooding and dewatering with application to Great Bay, New Hampshire. Estuar Coast Shelf Sci 47:119-141

Irish JL, Resio DT, Ratcliff JJ (2008) The influence of storm size on hurricane surge. J Phys Oceanogr 38(9):2003-2013. https://doi. org/10.1175/2008JPO3727.1

Jeschke A, Vater S, Behrens J (2017) A discontinuous Galerkin method for non-hydrostatic shallow water flows. Springer International Publishing, Cham, pp 247-255
Kesserwani G, Liang Q (2012) Locally limited and fully conserved RKDG2 shallow water solutions with wetting and drying. J Sci Comput 50(1):120-144. https://doi.org/10.1007/s10915-011-9476-4

Kopera MA, Giraldo FX (2014) Analysis of adaptive mesh refinement for IMEX discontinuous Galerkin solutions of the compressible Euler equations with application to atmospheric simulations. J Comput Phys 275:92-117

Kubatko EJ, Dawson C, Westerink JJ (2008) Time step restrictions for Runge-Kutta discontinuous Galerkin methods on triangular grids. J Comput Phys 227(23):9697-9710. https://doi.org/10.1016/j.jcp. 2008.07.026 URL http://www.sciencedirect.com/science/article/pii/ S00219991080 03963

Kubatko E, Yeager B, Ketcheson DI (2014) Optimal strong-stabilitypreserving Runge-Kutta time discretizations for discontinuous Galerkin methods. J Sci Comput 60(2):313-344. https://doi.org/ 10.1007/s10915-013-9796-7

Large WG, Pond S (1981) Open Ocean momentum flux measurements in moderate to strong winds. J Phys Oceanogr 11(3):324-336. https:// doi.org/10.1175/1520-0485(1981)011<0324:OOMFMI>2.0.CO;2

LeVeque RJ (2002) Finite volume methods for hyperbolic problems. Cambridge texts in applied mathematics. Cambridge University Press. https://doi.org/10.1017/CBO9780511791253

Lin N, Emanuel KA, Smith JA, Vanmarcke E (2010) Risk assessment of hurricane storm surge for New York City. J Geophys Res Atmos 115(D18). https://doi.org/10.1029/2009JD013630 URL https:// agupubs.onlinelibrary.wiley.com/doi/abs/10.1029/2009J D013630

Luettich R, Westerink J (1995) Implementation and testing of elemental flooding and drying in the ADCIRC hydrodynamic model. Tech. Rep., Department Of The Army, coastal engineering research center, waterways Experiment Station, US Army Corps of Engineers. Final Contractors Report

Mandli KT (2011) Finite volume methods for the multilayer shallow water equations with applications to storm surges. Ph.D. thesis, University of Washington

Mandli KT, Dawson CN (2014) Adaptive mesh refinement for storm surge. Ocean Model 75:36-50

Marras, S., Kopera, M.A., Constantinescu, E.M., Suckale, J., Giraldo, F.X.: A continuous/discontinuous Galerkin solution of the shallow water equations with dynamic viscosity, high-order wetting and drying, and implicit time integration (2016)

Piggott MD, Gorman GJ, Pain CC, Allison PA, Candy AS, Martin BT, Wells MR (2008a) A new computational framework for multi-scale ocean modelling based on adapting unstructured meshes. Int $\mathrm{J}$ Numer Methods Fluids 56(8):1003-1015

Piggott MD, Pain CC, Gorman GJ, Marshall DP, Killworth PD (2008b) Unstructured adaptive meshes for ocean modeling. In: Hasumi $\mathrm{H}$, Hecht MW (eds) Ocean modeling in an Eddying Regime. AGU, pp 383-408. https://doi.org/10.1029/177GM22

Powell MD (2007) New findings on hurricane intensity, wind field extent, and surface drag coefficient behavior. Tech. Rep., National Oceanic and Atmospheric Administration. Technical Report

Qiang J, Liang Q, Wang G, Zheng J (2016) Testing a shock-capturing hydrodynamic model for storm surge simulation. Proceedings of the Twenty-sixth (2016) International Ocean and Polar Engineering Conference Rhodes, Greece pp 747-753

Rivara MC (1984) Algorithms for refining triangular grids suitable for adaptive and multigrid techniques. Int J Numer Methods Eng 20:745-756

Sanay R, Valle-Levinson A (2005) Wind-induced circulation in semienclosed homogeneous, rotating basins. J Phys Oceanogr 35:2520-2531

Toro EF (2009) Riemann solvers and numerical methods for fluid dynamics: a practical introduction. Springer

Tull N (2018) Improving accuracy of real-time storm surge inundation predictions. Master's thesis, North Carolina State University, Raleigh, North Carolina

Vater S, Beisiegel N, Behrens J (2015) A limiter-based well-balanced discontinuous Galerkin method for shallow-water flows with 
wetting and drying: one-dimensional case. Adv Water Resour 85:113. https://doi.org/10.1016/j.advwatres.2015.08.008

Vater S, Beisiegel N, Behrens J (2019) A limiter-based well-balanced discontinuous Galerkin method for shallow-water flows with wetting and drying: triangular grids. Int $\mathrm{J}$ Numer Methods Fluids. https://doi.org/10.1002/fld.4762 In print
Weisberg RH, Zheng L (2006) Hurricane storm surge simulations for Tampa Bay. Estuar Coasts 29(6A):899-913

Xu Z, Chen XY, Liu Y (2014) A new Runge-Kutta discontinuous Galerkin method with conservation constraint to improve CFL condition for solving conservation laws. J Comput Phys 278:348-377. https://doi.org/10.1016/j.jcp.2014.08.042 URL http://www. sciencedirect.com/science/article/pii/S00219991140 06184 